\title{
Two-loop scalar kinks
}

\author{
Jarah Evslin $\oplus^{1,2, *}$ and Hengyuan Guo $\oplus^{1,2, \dagger}$ \\ ${ }^{1}$ Institute of Modern Physics, NanChangLu 509, Lanzhou 730000, China \\ ${ }^{2}$ University of the Chinese Academy of Sciences, YuQuanLu 19A, Beijing 100049, China
}

(Received 20 March 2021; accepted 14 May 2021; published 15 June 2021)

\begin{abstract}
At one loop, quantum kinks are described by a sum of quantum harmonic oscillator Hamiltonians, and the ground state is just the product of the oscillator ground states. Two-loop kink masses are only known in integrable and supersymmetric cases and two-loop states have never been found. We find the two-loop kink mass and explicitly construct the two-loop kink ground state in a scalar field theory with an arbitrary nonderivative potential. We use a coherent state operator that maps the vacuum sector to the kink sector, allowing all states to be treated with a single Hamiltonian that needs to be renormalized only once, eliminating the need for regulator matching conditions. Our calculation is greatly simplified by a recently introduced alternative to collective coordinates, in which the kink momentum is fixed perturbatively.
\end{abstract}

DOI: 10.1103/PhysRevD.103.125011

\section{INTRODUCTION}

Quantum solitons at strong coupling are poorly understood, and yet are widely believed to be somehow responsible for confinement in Yang-Mills and QCD. Understanding them is therefore of critical importance. However we would like to suggest that this is premature as solitons at weak coupling are also not understood.

Early papers on quantum solitons produced consistent results. Beginning with the pioneering paper [1], one-loop corrections to kink masses were calculated by introducing a vacuum sector and a kink sector Hamiltonian, regularizing them both, identifying the regulators and renormalizing. In the 1970s, the regulator was a cutoff in the number of modes. In the 1980s, authors instead calculated one-loop corrections to the masses of supersymmetric kinks, regularizing with an energy cutoff. It was only in the following decade that Ref. [2] reported that, when applied to the same kink, these two methods yielded different masses.

The basic problem is as follows. A theory is defined by its Hamitonian together with a regulator and renormalization scheme. One thus expects masses to depend on these three choices. However, once these are fixed, the theory is fixed as are all observables. In particular, nothing may depend on an arbitrary choice of matching conditions for

\footnotetext{
jarah@impcas.ac.cn

guohengyuan@impcas.ac.cn
}

Published by the American Physical Society under the terms of the Creative Commons Attribution 4.0 International license. Further distribution of this work must maintain attribution to the author(s) and the published article's title, journal citation, and DOI. Funded by SCOAP ${ }^{3}$. regulators. At most one such inequivalent choice may be correct, but which?

Many responses to this question have since appeared in the literature. The most common interpretation is that some regulator matching conditions give answers which are "bad" [3], and so either different regulators should be used such as in Ref. [1] or different methods, such as that of Ref. [4], which yields the correct soliton mass at one loop. Another response is that the problem is caused by linear divergences, but these may be made logarithmic by taking a derivative with respect to a mass scale and then integrating using a physical principle to fix the constant of integration [5]. This strategy has been successfully employed to reproduce the two-loop mass of the Sine-Gordon soliton. However, as noted by an overlapping collection of authors in [6], this strategy fails with some choices of boundary conditions and, more importantly, it does not shed light on which matching conditions should be allowed. Perhaps the most interesting suggestion, proposed in Ref. [6], is that an ultraviolet cutoff may only be imposed if the nontrivial background itself has no effect above that cutoff. It is an appealing physical principle; however, in practice it does not entirely determine how the density of states is to be corrected. Ultimately the authors chose this correction to reproduce the known answer, leading one to wonder just what prescription works when the answer is not already known. Later it was proposed [7] that instead the matching condition should keep the same mode density in every sector. However the authors note that this proposal is only expected to work at one loop.

This state of affairs has motivated our program to systematically study perturbation theory about quantum solitons in a formalism with no matching conditions. Instead, following [8], we introduce a nonlocal operator 
TABLE I. Summary of notation.

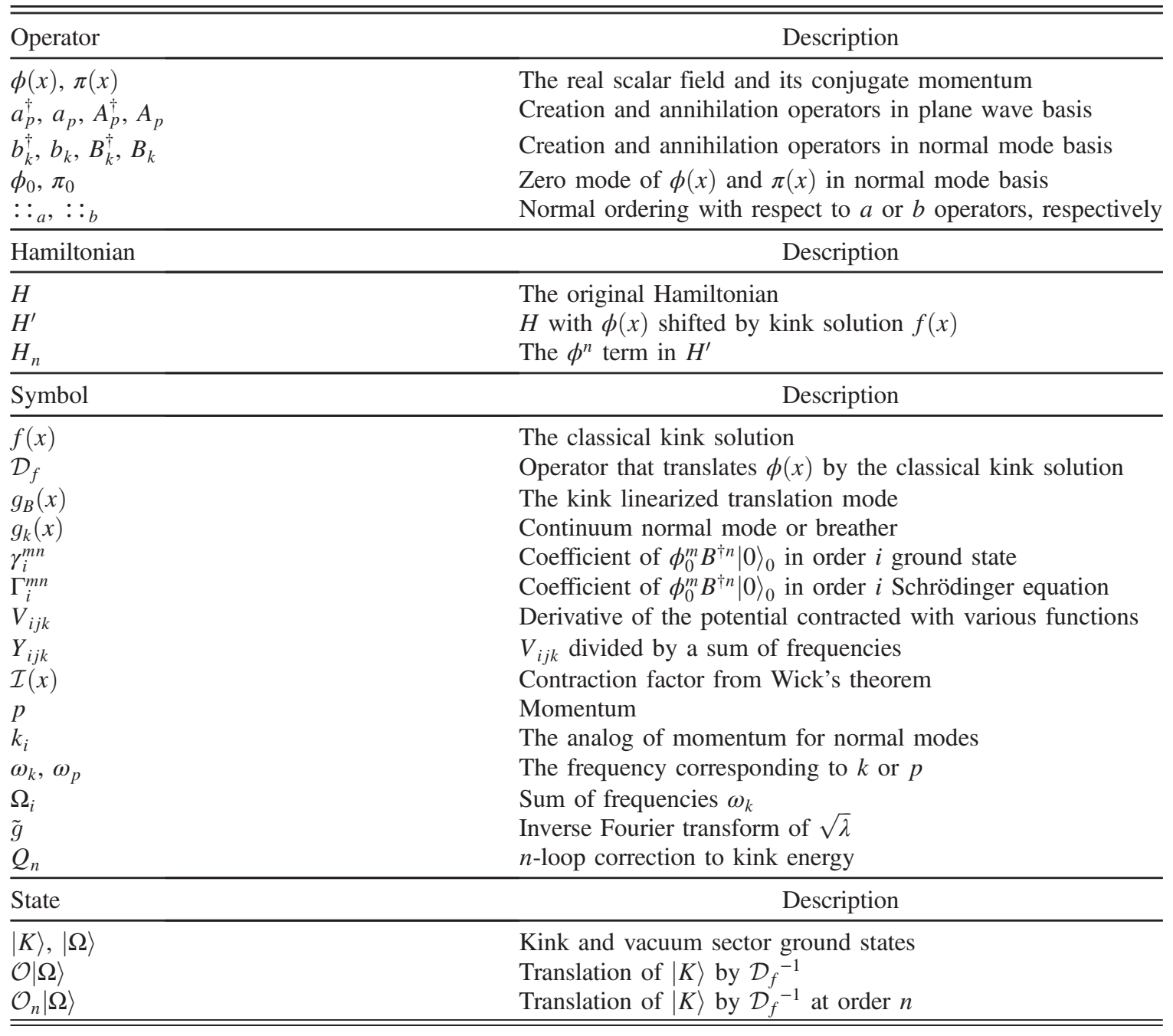

which maps the vacuum sector to the one soliton sector. ${ }^{1}$ This allows all computations involving both sectors to be performed using the original Hamiltonian, with no need to introduce another Hamiltonian for the soliton sector. We thus need to renormalize only once, obviating the need for regulator matching. In Refs. $[10,11]$ this was carried out at one loop in the $1+1 \mathrm{~d} \phi^{4}$ and Sine-Gordon models. At one loop these results were known as the theory is free. The first correction to the states was reported in Ref. [12]. The present paper continues to two loops, for a general scalar kink in $1+1$ dimensions. The kink ground state and mass are found.

We begin in Sec. II with a review of our formalism. Then we calculate the two-loop quantum ground states in two steps. Our states are decomposed in a power series in the zero mode $\phi_{0}$ of the scalar field. We refer to the constant terms in this decomposition as $\phi_{0}$ primaries and others as $\phi_{0}$ descendants. In Sec. III we use translation invariance to fix all $\phi_{0}$ descendants in terms of $\phi_{0}$ primaries. Next, in

\footnotetext{
${ }^{1}$ For a computationally similar approach without the nonlocal operator, see Ref. [9].
}

Sec. IV, we use Schrödinger's equation to find the $\phi_{0}$ primaries. As an application, in Sec. V we present a formula for the two-loop mass correction to kinks in $(1+1)$-dimensional scalar theories with an arbitrary potential. In Appendix A we show that the states that we have constructed indeed solve Schrödinger's equation.

\section{REVIEW}

In this section we will review the formalism for treating quantum kinks presented in Refs. [10,13,14]. Table I summarizes some of our notation.

Let $\phi(x)$ and $\pi(x)$ be a Schrödinger picture real scalar field and its conjugate in $1+1$ dimensions, whose dynamics are described by the Hamiltonian

$$
\begin{aligned}
H= & \int d x \mathcal{H}(x), \\
\mathcal{H}(x)= & \frac{1}{2}: \pi(x) \pi(x):_{a}+\frac{1}{2}: \partial_{x} \phi(x) \partial_{x} \phi(x):_{a} \\
& +\frac{M^{2}}{\lambda}: \mathcal{V}[\sqrt{\lambda} \phi(x)]:{ }_{a} .
\end{aligned}
$$


Here $M$ and $\sqrt{\lambda}$ have dimensions of mass and action ${ }^{-1 / 2}$, respectively. We expand in $\lambda \hbar$ and set $\hbar=1$. Also we will define the dimensionful potential

$$
V=M^{2} \mathcal{V}
$$

The normal ordering $::_{a}$ is defined below. We remind the reader that in $1+1$ dimensions, normal ordering is sufficient to render scalar field theories UV finite.

If $V$ has degenerate minima, then there will be a classical kink solution

$$
\phi(x, t)=f(x) .
$$

We normalize $M$ such that $\mathcal{V}^{\prime \prime}$ evaluated at both minima appearing at the end of the kink is equal to unity, which requires the simplifying assumption that these are equal. In the Schrödinger picture, where we will always work, the displacement operator

$$
\mathcal{D}_{f}=\exp \left(-i \int d x f(x) \pi(x)\right)
$$

satisfies [10]

$: F[\pi(x), \phi(x)]:{ }_{a} \mathcal{D}_{f}=\mathcal{D}_{f}: F[\pi(x), \phi(x)+f(x)]:{ }_{a}$,

where $F$ is an arbitrary functional. This operator takes the vacuum sector to the kink sector. In particular one may relate the ground states $|\Omega\rangle$ and $|K\rangle$ of the two respective sectors

$$
|K\rangle=\mathcal{D}_{f} \mathcal{O}|\Omega\rangle
$$

using the perturbative operator $\mathcal{O}$. The kink ground state $|K\rangle$ is an eigenstate of the Hamiltonian $H$ and so $\mathcal{O}|\Omega\rangle$ must be an eigenstate of the Hamiltonian

$$
\begin{aligned}
H^{\prime}=\mathcal{D}_{f}^{-1} H \mathcal{D}_{f} & =Q_{0}+H_{2}+H_{I} \\
H_{2} & =\frac{1}{2} \int d x\left[: \pi^{2}(x):_{a}+:\left(\partial_{x} \phi(x)\right)^{2}:_{a}+V^{\prime \prime}[\sqrt{\lambda} f(x)]: \phi^{2}(x):_{a}\right] .
\end{aligned}
$$

Here $Q_{0}$ is the classical mass of the solution $f(x)$ and $H_{I}$ contains all higher order terms in $\sqrt{\lambda}$.

The free Hamiltonian $\mathrm{H}_{2}$ leads to classical linear equations of motion whose constant frequency solutions are the normal modes $g(x)$ of the kink

$$
\phi(x, t)=e^{-i \omega t} g(x), \quad V^{\prime \prime}[\sqrt{\lambda} f(x)] g(x)=\omega^{2} g(x)+g^{\prime \prime}(x) .
$$

There will be continuum solutions $g_{k}(x)$ labeled by an index $k$ such that ${ }^{2} \omega_{k}=\sqrt{M^{2}+k^{2}}$, breathers, and a single Goldstone mode $g_{B}(x)$

$$
g_{B}(x)=\frac{1}{\sqrt{Q_{0}}} f^{\prime}(x)
$$

with $\omega_{B}=0$. For brevity of notation, we will not distinguish between continuum solutions and breathers, and so it will be implicit that integrals over the continuous variable $k$ include a sum over the breathers.

We adopt the normalization conditions

$$
\int d x g_{k_{1}}(x) g_{k_{2}}^{*}(x)=2 \pi \delta\left(k_{1}-k_{2}\right), \quad \int d x\left|g_{B}(x)\right|^{2}=1,
$$

\footnotetext{
${ }^{2}$ The sign of $k$ is chosen to agree with the momentum of the corresponding plane wave at $|x| \gg 0$.
}

and we choose the phases such that

$$
g_{k}(-x)=g_{k}^{*}(x)=g_{-k}(x) .
$$

We also define inverse Fourier transforms

$$
\tilde{g}(p)=\int d x g(x) e^{i p x}
$$

satisfying the completeness relations

$\tilde{g}_{B}(p) \tilde{g}_{B}(q)+\int \frac{d k}{2 \pi} \tilde{g}_{k}(p) \tilde{g}_{-k}(q)=2 \pi \delta(p+q)$.

The same quantum field and its conjugate may be expanded in terms of plane waves

$$
\begin{aligned}
\phi(x) & =\int \frac{d p}{2 \pi} \frac{1}{\sqrt{2 \omega_{p}}}\left(a_{p}^{\dagger}+a_{-p}\right) e^{-i p x}, \\
\omega_{p} & =\sqrt{M^{2}+p^{2}} \\
\pi(x) & =i \int \frac{d p}{2 \pi} \sqrt{\frac{\omega_{p}}{2}}\left(a_{p}^{\dagger}-a_{-p}\right) e^{-i p x}
\end{aligned}
$$

or normal modes 


$$
\begin{array}{rlrl}
\phi(x) & =\phi_{B}(x)+\phi_{C}(x), & \pi(x)=\pi_{B}(x)+\pi_{C}(x), \\
\phi_{B}(x) & =\phi_{0} g_{B}(x), & \phi_{C}(x) & =\int \frac{d k}{2 \pi} \frac{1}{\sqrt{2 \omega_{k}}}\left(b_{k}^{\dagger}+b_{-k}\right) g_{k}(x), \\
\pi_{B}(x) & =\pi_{0} g_{B}(x), & \pi_{C}(x) & =i \int \frac{d k}{2 \pi} \sqrt{\frac{\omega_{k}}{2}}\left(b_{k}^{\dagger}-b_{-k}\right) g_{k}(x) .
\end{array}
$$

We define the plane wave normal ordering $::_{a}$ by placing the $a^{\dagger}$ to the left of the $a$ and normal mode normal ordering $::_{b}$ by placing $b^{\dagger}$ and $\phi_{0}$ to the left of $b$ and $\pi_{0}$.

Using the canonical algebra satisfied by $\phi(x)$ and $\pi(x)$ together with the completeness of the solutions [12]

$$
g_{B}(x) g_{B}(y)+\int \frac{d k}{2 \pi} g_{k}(x) g_{-k}(y)=\delta(x-y),
$$

one finds

$$
\left[a_{p}, a_{q}^{\dagger}\right]=2 \pi \delta(p-q), \quad\left[\phi_{0}, \pi_{0}\right]=i, \quad\left[b_{k_{1}}, b_{k_{2}}^{\dagger}\right]=2 \pi \delta\left(k_{1}-k_{2}\right) .
$$

These allow the plane wave normal ordered $\mathrm{H}_{2}$ to be rewritten in terms of a normal mode normal ordered free Hamiltonian plus a constant $Q_{1}$, which is the one-loop correction to the kink mass. This can be achieved one term a time

$$
\begin{aligned}
& : \pi_{B}^{2}(x):_{a}=: \pi_{B}^{2}(x):_{b}+g_{B}(x) \hat{\hat{g}}_{B}(x), \quad \hat{\hat{g}}_{B}(x)=-\int \frac{d p}{2 \pi} e^{-i x p} \frac{\omega_{p}}{2} \tilde{g}_{B}(p) \\
& : \pi_{C}^{2}(x):_{a}=: \pi_{C}^{2}(x):_{b}+\int \frac{d k}{2 \pi} g_{k}(x) \hat{\hat{g}}_{-k}(x), \quad \hat{\hat{g}}_{k}(x)=\int \frac{d p}{2 \pi} e^{-i x p}\left(\frac{\omega_{k}-\omega_{p}}{2}\right) \tilde{g}_{k}(p) \\
& : \phi_{B}^{2}(x):_{a}=: \phi_{B}^{2}(x):_{b}+g_{B}(x) \hat{g}_{B}(x), \quad \hat{g}_{B}(x)=-\int \frac{d p}{2 \pi} e^{-i x p} \frac{1}{2 \omega_{p}} \tilde{g}_{B}(p) \\
& : \phi_{C}^{2}(x):_{a}=: \phi_{C}^{2}(x):_{b}+\int \frac{d k}{2 \pi} g_{k}(x) \hat{g}_{-k}(x), \quad \hat{g}_{k}(x)=\int \frac{d p}{2 \pi} e^{-i x p}\left(\frac{1}{2 \omega_{k}}-\frac{1}{2 \omega_{p}}\right) \tilde{g}_{k}(p) .
\end{aligned}
$$

Applying the classical equations of motion (2.8) one finds

$$
\begin{aligned}
& V^{\prime \prime}[\sqrt{\lambda} f(x)]: \phi_{B}^{2}(x):_{a}=V^{\prime \prime}[\sqrt{\lambda} f(x)]: \phi_{B}^{2}(x):_{b}+g_{B}^{\prime \prime}(x) \hat{g}_{B}(x), \\
& V^{\prime \prime}[\sqrt{\lambda} f(x)]: \phi_{C}^{2}(x):_{a}=V^{\prime \prime}[\sqrt{\lambda} f(x)]: \phi_{C}^{2}(x):_{b}+\int \frac{d k}{2 \pi}\left(\omega_{k}^{2} g_{k}(x)+g_{k}^{\prime \prime}(x)\right) \hat{g}_{-k}(x) .
\end{aligned}
$$

The $g^{\prime \prime}$ terms cancel $: \partial \phi(x) \partial \phi(x):_{a}-: \partial \phi(x) \partial \phi(x):_{b}$ after an integration by parts, leaving

$$
\begin{aligned}
& H_{2}=Q_{1}+\frac{\pi_{0}^{2}}{2}+\int \frac{d k}{2 \pi} \omega_{k} b_{k}^{\dagger} b_{k}, \\
& Q_{1}=-\frac{1}{4} \int \frac{d k}{2 \pi} \int \frac{d p}{2 \pi} \frac{\left(\omega_{p}-\omega_{k}\right)^{2}}{\omega_{p}} \tilde{g}_{k}^{2}(p)-\frac{1}{4} \int \frac{d p}{2 \pi} \omega_{p} \tilde{g}_{B}(p) \tilde{g}_{B}(p) .
\end{aligned}
$$

We perform a semiclassical expansion of the kink ground state ${ }^{3}$ in powers of $\sqrt{\lambda}$

$$
\mathcal{O}|\Omega\rangle=\sum_{i=0}^{\infty}|0\rangle_{i} .
$$

The one-loop kink ground state $|0\rangle_{0}$ is a product of free vacua

\footnotetext{
${ }^{3}$ The $n$-loop ground state is the sum up to $i=2 n-2$. Note that there is no tree-level term in our expansion. In a sense made precise in Ref. [12], the tree-level ground state $|\Omega\rangle$ is automatically included in the one-loop $|0\rangle_{0}$ by the condition (2.21). Beginning the expansion with a tree-level ground state at a fixed center of mass would lead to an infinite first correction [15].
} 


$$
\pi_{0}|0\rangle_{0}=b_{k}|0\rangle_{0}=0 \text {. }
$$

In Ref. [16] we found a general Wick's formula for the conversion of plane wave to normal mode normal ordering. For powers of $\phi(x)$ it reads

$$
: \phi^{n}(x):_{a}=\sum_{m=0}^{\left\lfloor\frac{n}{2}\right\rfloor} \frac{n !}{2^{m} m !(n-2 m) !} \mathcal{I}^{m}(x): \phi^{n-2 m}(x):_{b},
$$

where

$$
\begin{aligned}
\mathcal{I}(x) & =g_{B}(x) \hat{g}_{B}(x)+\int \frac{d k}{2 \pi} g_{-k}(x) \hat{g}_{k}(x), \\
\hat{g}_{B}(x) & =-\int \frac{d p}{2 \pi} e^{-i p x} \frac{\tilde{g}_{B}(p)}{2 \omega_{p}}, \quad \hat{g}_{k}(x)=\int \frac{d p}{2 \pi} e^{-i p x} \tilde{g}_{k}(p)\left(\frac{1}{2 \omega_{k}}-\frac{1}{2 \omega_{p}}\right) .
\end{aligned}
$$

Using the completeness relations $(2.13)$ one can show $[12,16]$ that $\mathcal{I}(x)$ is determined by

$$
\partial_{x} \mathcal{I}(x)=\int \frac{d k}{2 \pi} \frac{1}{2 \omega_{k}} \partial_{x}\left|g_{k}(x)\right|^{2}
$$

together with the condition that it vanish at spatial infinity.

\section{TRANSLATION INVARIANCE}

In this section we will calculate the translation operator that acts on our states $\mathcal{O}|\Omega\rangle$ and will use it to fix all $\phi_{0}$ descendants (components of states that include operators $\left.\phi_{0}\right)$.

\section{A. The translation operator}

Let us define the shorthand

$$
\Delta_{i j}=\int d x g_{i}(x) g_{j}^{\prime}(x)=i \int \frac{d p}{2 \pi} p \tilde{g}_{i}(p) \tilde{g}_{j}(-p),
$$

where $i$ and $j$ may be a bound state or a momentum $k$. Note that $\Delta$ is antisymmetric. We will use reweighted creation and annihilation operators

$$
B_{k}^{\dagger}=\frac{b_{k}^{\dagger}}{\sqrt{2 \omega_{k}}}, \quad B_{k}=\sqrt{2 \omega_{k}} b_{k},
$$

which satisfy the same Heisenberg commutation relations as $b^{\dagger}$ and $b$.

The identity

$$
P \mathcal{D}_{f}=\mathcal{D}_{f}\left(P-\sqrt{Q_{0}} \pi_{0}\right)
$$

implies that the translation invariance

$$
P|K\rangle=P \mathcal{D}_{f} \sum_{i}|0\rangle_{i}=0
$$

is equivalent to

$$
P|0\rangle_{i}=\sqrt{Q_{0}} \pi_{0}|0\rangle_{i+1} .
$$

Our strategy will be to solve this equation by inverting $\pi_{0}$. Thus translation invariance fixes our states entirely up to an element of the kernel of $\pi_{0}$. We then only use the Schrödinger equation to fix the element of the kernel of $\pi_{0}$, thus greatly simplifying the problem. Note that the kernel of $\pi_{0}$ consists precisely of the $\phi_{0}$-primary states.

Let us write the translation operator as

$$
\begin{aligned}
P & =-\int d x \pi(x) \partial_{x} \phi(x), \\
& =-\int d x\left[\pi_{0} g_{B}(x) \int \frac{d k}{2 \pi} \phi_{k} g_{k}^{\prime}(x)+\left(\int \frac{d k}{2 \pi} \pi_{k} g_{k}(x)\right) \phi_{0} g_{B}^{\prime}(x)+\int \frac{d^{2} k}{(2 \pi)^{2}} \pi_{k_{1}} \phi_{k_{2}} g_{k_{1}}(x) g_{k_{2}}^{\prime}(x)\right], \\
& =\int \frac{d k}{2 \pi} \Delta_{k B}\left[i \phi_{0}\left(-\omega_{k} B_{k}^{\dagger}+\frac{B_{-k}}{2}\right)+\pi_{0}\left(B_{k}^{\dagger}+\frac{B_{-k}}{2 \omega_{k}}\right)\right]+i \int \frac{d^{2} k}{(2 \pi)^{2}} \Delta_{k_{1} k_{2}}\left(-\omega_{k_{1}} B_{k_{1}}^{\dagger} B_{k_{2}}^{\dagger}+\frac{B_{-k_{1}} B_{-k_{2}}}{4 \omega_{k_{2}}}-\frac{1}{2}\left(1+\frac{\omega_{k_{1}}}{\omega_{k_{2}}}\right) B_{k_{1}}^{\dagger} B_{-k_{2}}\right),
\end{aligned}
$$


and expand the $i$ th order kink ground state as

$$
|0\rangle_{i}=\sum_{m, n=0}^{\infty}|0\rangle_{i}^{m n}, \quad|0\rangle_{i}^{m n}=Q_{0}^{-i / 2} \int \frac{d^{n} k}{(2 \pi)^{n}} \gamma_{i}^{m n}\left(k_{1} \cdots k_{n}\right) \phi_{0}^{m} B_{k_{1}}^{\dagger} \cdots B_{k_{n}}^{\dagger}|0\rangle_{0} .
$$

We will refer to $m=0$ states or matrix elements $\gamma_{i}^{0 n}$ as $\phi_{0}$ primary and $m>0$ states as $\phi_{0}$ descendants. Then the translation invariance (3.5) yields the recursion relation

$$
\begin{aligned}
\gamma_{i+1}^{m n}\left(k_{1} \cdots k_{n}\right)= & \Delta_{k_{n} B}\left(\gamma_{i}^{m, n-1}\left(k_{1} \cdots k_{n-1}\right)+\frac{\omega_{k_{n}}}{m} \gamma_{i}^{m-2, n-1}\left(k_{1} \cdots k_{n-1}\right)\right) \\
& +\int \frac{d k^{\prime}}{2 \pi} \Delta_{-k^{\prime} B} \sum_{j=0}^{n}\left(\frac{\gamma_{i}^{m, n+1}\left(k_{1} \cdots k_{j}, k^{\prime}, k_{j+1} \cdots k_{n}\right)}{2 \omega_{k^{\prime}}}-\frac{\gamma_{i}^{m-2, n+1}\left(k_{1} \cdots k_{j}, k^{\prime}, k_{j+1} \cdots k_{n}\right)}{2 m}\right) \\
& +\frac{1}{2 m} \sum_{j=1}^{n} \int \frac{d k^{\prime}}{2 \pi} \Delta_{k_{n},-k^{\prime}}\left(1+\frac{\omega_{k_{n}}}{\omega_{k^{\prime}}}\right) \gamma_{i}^{m-1, n}\left(k_{1} \cdots k_{j-1}, k^{\prime}, k_{j} \cdots k_{n-1}\right)+\frac{\omega_{k_{n-1}} \Delta_{k_{n-1} k_{n}}}{m} \gamma_{i}^{m-1, n-2}\left(k_{1} \cdots k_{n-2}\right) \\
& -\int \frac{d^{2} k^{\prime}}{(2 \pi)^{2}} \frac{\Delta_{-k_{1}^{\prime},-k_{2}^{\prime}}}{2 m \omega_{k_{2}^{\prime}}} \sum_{j_{1}=1}^{n+1} \sum_{j_{2}=j_{1}+1}^{n+2} \gamma_{i}^{m-1, n+2}\left(k_{1} \cdots k_{j_{1}-1}, k_{1}^{\prime}, k_{j_{1}} \cdots k_{j_{2}-2}, k_{2}^{\prime}, k_{j_{2}-1} \cdots k_{n}\right) .
\end{aligned}
$$

This recursion relation determines all $\phi_{0}$ descendants in terms of $\phi_{0}$-primary states plus the free state corresponding to the one-loop initial condition $\gamma_{0}$. It does not determine the $\phi_{0}$ primaries, as it corresponds to a particular solution of Eq. (3.5) and the addition of any element of the kernel of $\pi_{0}$, in other words any $\phi_{0}$-primary state, is another solution.

In general this recursion relation leads to infrared (IR) divergences. In Ref. [17], two kinds of IR divergences are identified. The first results from singularities in $\Delta_{k_{1} k_{2}}$ as $k_{1}+k_{2}$ tends to zero [see for example Eq. (5.18)] and, in that case, describes the recoil momentum of a kink when a normal mode is excited in the center of mass frame. The second results from divergences in the initial conditions $\gamma_{0}$, for example if one begins with an excited isolated continuum normal mode. These divergences correspond to interactions that do not involve the excited mode. In both cases the divergences must in general be kept to arrive at the correct final answer. Realistic initial conditions for excited states are wave packets that depend smoothly on the continuum $k$ and we do not know whether such IR divergences are avoided at all orders in that case. In the present paper, we are interested in the ground state and so $\gamma_{0}$ is independent of $k$ and we have no recoil. In this case no IR divergences appear to the order calculated below, although there are terms of the form $\left(\omega_{k_{1}}-\omega_{k_{2}}\right) \delta\left(k_{1}-\right.$ $k_{2}$ ) in which divergences are avoided by the structure of the coefficients.

\section{B. Constructing translation-invariant states}

At one loop, the quantum kink is described by a series of harmonic oscillators and so its spectrum is known precisely [1]. To find a Hamiltonian eigenstate at higher but finite order, one need only start the recursion (3.8) at $i=0$ with the one-loop avatar of the state of interest.

In this note we will apply this strategy to the ground state, corresponding to the initial condition

$$
\gamma_{0}^{m n}=\delta_{m 0} \delta_{n 0} \gamma_{0}^{00}
$$

The first recursion is depicted in the left panel of Fig. 1, where it determines the squares in terms of the star, which corresponds to the initial condition. More precisely, it yields

$\gamma_{1}^{12}\left(k_{1}, k_{2}\right)=\omega_{k_{1}} \Delta_{k_{1} k_{2}} \gamma_{0}^{00}, \quad \gamma_{1}^{21}\left(k_{1}\right)=\frac{\omega_{k_{1}} \Delta_{k_{1}} B}{2} \gamma_{0}^{00}$.

We are not interested in calculating the $\phi_{0}$ primaries ( $m=0$ terms) because these are in the kernel of $\pi_{0}$, and so they are

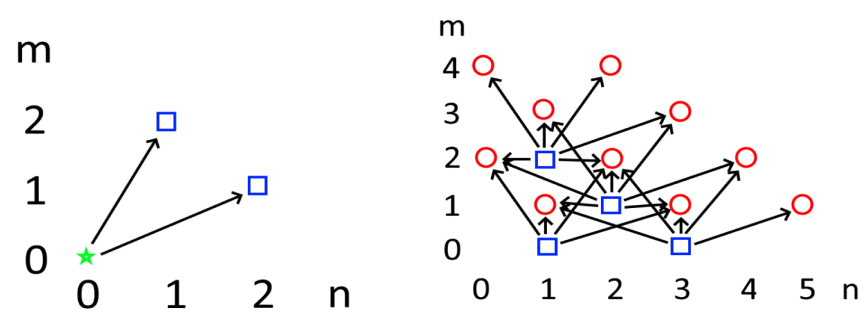

FIG. 1. The $\gamma_{i}^{m n}$ generated by the recursion relation at $i=1$ (left) and $i=2$ (right). Green stars, blue squares and red circles represent elements at $i=0, i=1$, and $i=2$, respectively. As $\phi_{0}$ primaries ( $m=0$ elements) are in the kernel of $\pi_{0}$, they are not fixed by (3.5) and so arrows to such elements are not shown. 
not determined by translation invariance. These will be calculated using Schrödinger's equation in Sec. IV.

We may continue by simply plugging in to our recursion relation (3.8). But we can simplify things somewhat by noticing that (3.7) does not completely determine the functions $\gamma_{i}^{m n}\left(k_{1} \cdots k_{n}\right)$. For example, one may add any function that is antisymmetric under the exchange of any $k_{i}$ and $k_{j}$ without affecting $|0\rangle$. Therefore we are free to symmetrize each function. As this will simplify our answer, that will be our convention: it will be understood that after calculating each $\gamma$ using (3.8) it should be symmetrized before the next recursion. This convention allows one to perform all of the sums in our recursion relation (3.8), leaving

$$
\begin{aligned}
\gamma_{i+1}^{m n}\left(k_{1} \cdots k_{n}\right)= & \Delta_{k_{n} B}\left(\gamma_{i}^{m, n-1}\left(k_{1} \cdots k_{n-1}\right)+\frac{\omega_{k_{n}}}{m} \gamma_{i}^{m-2, n-1}\left(k_{1} \cdots k_{n-1}\right)\right) \\
& +(n+1) \int \frac{d k^{\prime}}{2 \pi} \Delta_{-k^{\prime} B}\left(\frac{\gamma_{i}^{m, n+1}\left(k_{1} \cdots k_{n}, k^{\prime}\right)}{2 \omega_{k^{\prime}}}-\frac{\gamma_{i}^{m-2, n+1}\left(k_{1} \cdots k_{n}, k^{\prime}\right)}{2 m}\right) \\
& +\frac{\omega_{k_{n-1}} \Delta_{k_{n-1}} k_{n}}{m} \gamma_{i}^{m-1, n-2}\left(k_{1} \cdots k_{n-2}\right)+\frac{n}{2 m} \int \frac{d k^{\prime}}{2 \pi} \Delta_{k_{n},-k^{\prime}}\left(1+\frac{\omega_{k_{n}}}{\omega_{k^{\prime}}}\right) \gamma_{i}^{m-1, n}\left(k_{1} \cdots k_{n-1}, k^{\prime}\right) \\
& -\frac{(n+2)(n+1)}{2 m} \int \frac{d^{2} k^{\prime}}{(2 \pi)^{2}} \frac{\Delta_{-k_{1}^{\prime},-k_{2}^{\prime}}}{2 \omega_{k_{2}^{\prime}}} \gamma_{i}^{m-1, n+2}\left(k_{1} \cdots k_{n}, k_{1}^{\prime}, k_{2}^{\prime}\right) .
\end{aligned}
$$

In summary, the recursion relation (3.8) always yields a correct $\gamma_{i+1}$ whereas the simpler (3.11) is also correct if one first symmetrizes each $\gamma_{i}^{m n}\left(k_{1} \cdots k_{n}\right)$ with respect to its arguments $k_{1} \cdots k_{n}$. Thus to apply (3.11) to derive $\gamma_{2}$ we must first symmetrize all $\gamma_{1}^{m n}$ with $n \geq 2$. We only found one such element, which, after symmetrizing using the antisymmetry of $\Delta$, becomes

$$
\gamma_{1}^{12}\left(k_{1}, k_{2}\right)=\frac{\left(\omega_{k_{1}}-\omega_{k_{2}}\right) \Delta_{k_{1} k_{2}}}{2} \gamma_{0}^{00}
$$

What about the $\phi_{0}$ primaries $\gamma_{1}^{0 n}$ ? These are not fixed by translation invariance as they are in the kernel of $\pi_{0}$. Rather they are determined using the Schrödinger equation. In a scalar theory with a canonical kinetic term, $\phi$ will have dimensions of $[\text { action }]^{1 / 2}$. As each $|0\rangle_{i}$ is suppressed by $\hbar^{1 / 2}$ with respect to $|0\rangle_{i-1}$, it may only depend on terms in the potential up to $\phi^{2+i}$. Therefore $|0\rangle_{1}$ and so $\gamma_{1}$ only depend on $\phi^{3}$ terms. As a result the only nonzero entries resulting from the Schrödinger equation can be $\gamma_{1}^{01}$ and $\gamma_{1}^{03}$.

Finally we are ready to apply (3.11) to calculate $\gamma_{2}$. Remember that the recursion relations only determine $\phi_{0}$ descendants $(m>0)$, so over all we expect $3,4,5$, and 6 contributions from $\gamma_{1}^{01}, \gamma_{1}^{03}, \gamma_{1}^{21}$, and $\gamma_{1}^{12}$, respectively. These are the circles in the right panel of Fig. 1. The $\gamma_{2}^{m n}$ corresponding to a circle at $(m, n)$ is a sum of terms, one for each arrow ending on that circle, which are each proportional to the $\gamma_{1}^{m^{\prime} n^{\prime}}$ found at the beginning of the corresponding arrow.

At $m=1$ we find

$$
\begin{aligned}
\gamma_{2}^{11}\left(k_{1}\right) & =\int \frac{d k^{\prime}}{2 \pi} \Delta_{-k^{\prime} B} \frac{\gamma_{1}^{12}\left(k_{1}, k^{\prime}\right)}{\omega_{k^{\prime}}}-\frac{3}{4} \int \frac{d^{2} k^{\prime}}{(2 \pi)^{2}} \frac{\Delta_{-k_{1}^{\prime},-k_{2}^{\prime}}}{\omega_{k_{2}^{\prime}}} \gamma_{1}^{03}\left(k_{1}, k_{1}^{\prime}, k_{2}^{\prime}\right)+\frac{1}{2} \int \frac{d k^{\prime}}{2 \pi} \Delta_{k_{1},-k^{\prime}}\left(1+\frac{\omega_{k_{1}}}{\omega_{k^{\prime}}}\right) \gamma_{1}^{01}\left(k^{\prime}\right), \\
& =\frac{1}{2} \int \frac{d k^{\prime}}{2 \pi}\left(\frac{\omega_{k_{1}}}{\omega_{k^{\prime}}}-1\right) \Delta_{k_{1} k^{\prime}} \Delta_{-k^{\prime} B} \gamma_{0}^{00}-\frac{3}{2} \int \frac{d^{2} k^{\prime}}{(2 \pi)^{2}} \frac{\Delta_{-k_{1}^{\prime},-k_{2}^{\prime}}}{\omega_{k_{2}^{\prime}}} \gamma_{1}^{03}\left(k_{1}, k_{1}^{\prime}, k_{2}^{\prime}\right)+\frac{1}{2} \int \frac{d k^{\prime}}{2 \pi} \Delta_{k_{1},-k^{\prime}}\left(1+\frac{\omega_{k_{1}}}{\omega_{k^{\prime}}}\right) \gamma_{1}^{01}\left(k^{\prime}\right),
\end{aligned}
$$

and 


$$
\begin{aligned}
\gamma_{2}^{13}\left(k_{1}, k_{2}, k_{3}\right) & =\omega_{k_{2}} \Delta_{k_{2} k_{3}} \gamma_{1}^{01}\left(k_{1}\right)+\Delta_{k_{3} B} \gamma_{1}^{12}\left(k_{1}, k_{2}\right)+\frac{3}{2} \int \frac{d k^{\prime}}{2 \pi} \Delta_{k_{3},-k^{\prime}}\left(1+\frac{\omega_{k_{3}}}{\omega_{k^{\prime}}}\right) \gamma_{1}^{03}\left(k_{1}, k_{2}, k^{\prime}\right), \\
& =\omega_{k_{2}} \Delta_{k_{2} k_{3}} \gamma_{1}^{01}\left(k_{1}\right)+\frac{1}{2} \Delta_{k_{3} B}\left(\omega_{k_{1}}-\omega_{k_{2}}\right) \Delta_{k_{1} k_{2}} \gamma_{0}^{00}+\frac{3}{2} \int \frac{d k^{\prime}}{2 \pi} \Delta_{k_{3},-k^{\prime}}\left(1+\frac{\omega_{k_{3}}}{\omega_{k^{\prime}}}\right) \gamma_{1}^{03}\left(k_{1}, k_{2}, k^{\prime}\right), \\
\gamma_{2}^{15}\left(k_{1} \cdots k_{5}\right) & =\omega_{k_{4}} \Delta_{k_{4} k_{5}} \gamma_{1}^{03}\left(k_{1}, k_{2}, k_{3}\right) .
\end{aligned}
$$

Next at $m=2$

$$
\begin{aligned}
\gamma_{2}^{20} & =\int \frac{d k^{\prime}}{2 \pi} \Delta_{-k^{\prime} B}\left(\frac{\gamma_{1}^{21}\left(k^{\prime}\right)}{2 \omega_{k^{\prime}}}-\frac{\gamma_{1}^{01}\left(k^{\prime}\right)}{4}\right)-\frac{1}{4} \int \frac{d^{2} k^{\prime}}{(2 \pi)^{2}} \frac{\Delta_{-k_{1}^{\prime},-k_{2}^{\prime}}}{\omega_{k_{2}^{\prime}}} \gamma_{1}^{12}\left(k_{1}^{\prime}, k_{2}^{\prime}\right) \\
& =\frac{1}{4} \int \frac{d k^{\prime}}{2 \pi} \Delta_{-k^{\prime} B}\left(\Delta_{k^{\prime} B} \gamma_{0}^{00}-\gamma_{1}^{01}\left(k^{\prime}\right)\right)+\frac{1}{8} \int \frac{d^{2} k^{\prime}}{(2 \pi)^{2}}\left(1-\frac{\omega_{k_{1}^{\prime}}}{\omega_{k_{2}^{\prime}}}\right) \Delta_{k_{1}^{\prime} k_{2}^{\prime}} \Delta_{-k_{1}^{\prime},-k_{2}^{\prime}} \gamma_{0}^{00},
\end{aligned}
$$

and

$$
\begin{aligned}
\gamma_{2}^{22}\left(k_{1}, k_{2}\right)= & \Delta_{k_{2} B}\left(\gamma_{1}^{21}\left(k_{1}\right)+\frac{\omega_{k_{2}}}{2} \gamma_{1}^{01}\left(k_{1}\right)\right)-\frac{3}{4} \int \frac{d k^{\prime}}{2 \pi} \Delta_{-k^{\prime} B} \gamma_{1}^{03}\left(k_{1}, k_{2}, k^{\prime}\right)+\frac{1}{2} \int \frac{d k^{\prime}}{2 \pi} \Delta_{k_{2},-k^{\prime}}\left(1+\frac{\omega_{k_{2}}}{\omega_{k^{\prime}}}\right) \gamma_{1}^{12}\left(k_{1}, k^{\prime}\right), \\
= & \frac{\Delta_{k_{2} B}}{2}\left(\omega_{k_{1}} \Delta_{k_{1} B} \gamma_{0}^{00}+\omega_{k_{2}} \gamma_{1}^{01}\left(k_{1}\right)\right)-\frac{3}{4} \int \frac{d k^{\prime}}{2 \pi} \Delta_{-k^{\prime} B} \gamma_{1}^{03}\left(k_{1}, k_{2}, k^{\prime}\right) \\
& +\frac{1}{4} \int \frac{d k^{\prime}}{2 \pi} \Delta_{k_{2},-k^{\prime}}\left(1+\frac{\omega_{k_{2}}}{\omega_{k^{\prime}}}\right)\left(\omega_{k_{1}}-\omega_{k^{\prime}}\right) \Delta_{k_{1} k^{\prime}} \gamma_{0}^{00}, \\
\gamma_{2}^{24}\left(k_{1} \cdots k_{4}\right)= & \frac{\omega_{k_{3}} \Delta_{k_{3} k_{4}}}{2} \gamma_{1}^{12}\left(k_{1}, k_{2}\right)+\Delta_{k_{4} B} \frac{\omega_{k_{4}}}{2} \gamma_{1}^{03}\left(k_{1} \cdots k_{3}\right), \\
= & \frac{\omega_{k_{1}} \omega_{k_{3}} \Delta_{k_{1} k_{2}} \Delta_{k_{3} k_{4}}}{2} \gamma_{0}^{00}+\frac{\omega_{k_{4}} \Delta_{k_{4} B}}{2} \gamma_{1}^{03}\left(k_{1} \cdots k_{3}\right) .
\end{aligned}
$$

Continuing to $m=3$ we find

$$
\begin{aligned}
\gamma_{2}^{31}\left(k_{1}\right) & =-\frac{1}{3} \int \frac{d k^{\prime}}{2 \pi} \Delta_{-k^{\prime} B} \gamma_{1}^{12}\left(k_{1}, k^{\prime}\right)+\frac{1}{6} \int \frac{d k^{\prime}}{2 \pi} \Delta_{k_{1},-k^{\prime}}\left(1+\frac{\omega_{k_{1}}}{\omega_{k^{\prime}}}\right) \gamma_{1}^{21}\left(k^{\prime}\right), \\
& =\frac{\gamma_{0}^{00}}{6} \int \frac{d k^{\prime}}{2 \pi}\left[\left(\omega_{k^{\prime}}-\omega_{k_{1}}\right) \Delta_{k_{1} k^{\prime}} \Delta_{-k^{\prime} B}+\frac{1}{2} \Delta_{k_{1},-k^{\prime}}\left(\omega_{k_{1}}+\omega_{k^{\prime}}\right) \omega_{k^{\prime}} \Delta_{k^{\prime} B}\right], \\
& =\gamma_{0}^{00} \int \frac{d k^{\prime}}{2 \pi}\left(\frac{\omega_{k^{\prime}}}{4}-\frac{\omega_{k_{1}}}{12}\right) \Delta_{k_{1} k^{\prime}} \Delta_{-k^{\prime} B}, \\
\gamma_{2}^{33}\left(k_{1}, k_{2}, k_{3}\right) & =\frac{\omega_{k_{3}} \Delta_{k_{3} B}}{3} \gamma_{1}^{12}\left(k_{1}, k_{2}\right)+\frac{\omega_{k_{2}} \Delta_{k_{2} k_{3}}}{3} \gamma_{1}^{21}\left(k_{1}\right), \\
& =\left(\omega_{k_{3}} \Delta_{k_{3} B}\left(\omega_{k_{1}}-\omega_{k_{2}}\right) \Delta_{k_{1} k_{2}}+\omega_{k_{2}} \Delta_{k_{2} k_{3}} \omega_{k_{1}} \Delta_{k_{1} B}\right) \frac{\gamma_{0}^{00}}{6} .
\end{aligned}
$$

Note that, since $\gamma_{2}^{33}$ is defined by its symmetric contraction with $B_{k_{1}}^{\dagger} B_{k_{2}}^{\dagger} B_{k_{3}}^{\dagger}$, one is free to add any term that is annihilated by the symmetrization of $k_{1}, k_{2}$, and $k_{3}$. Thus one may freely redefine

$$
\gamma_{2}^{33}\left(k_{1}, k_{2}, k_{3}\right)=\frac{\omega_{k_{1}} \Delta_{k_{1} B} \omega_{k_{2}} \Delta_{k_{2} k_{3}}}{2} \gamma_{0}^{00}
$$


In other words, different paths from $\gamma_{0}^{00}$ to $\gamma_{2}^{33}$ lead to contributions that are proportional. This suggests that to some extent it may be possible to explicitly solve our recursion formula. Finally the $m=4$ terms are

$$
\begin{aligned}
\gamma_{2}^{40} & =-\int \frac{d k^{\prime}}{2 \pi} \Delta_{-k^{\prime} B} \frac{\gamma_{1}^{21}\left(k^{\prime}\right)}{8}=\frac{\gamma_{0}^{00}}{16} \int \frac{d k^{\prime}}{2 \pi} \omega_{k^{\prime}} \Delta_{B k^{\prime}} \Delta_{-k^{\prime} B}, \\
\gamma_{2}^{42}\left(k_{1}, k_{2}\right) & =\Delta_{k_{2} B} \frac{\omega_{k_{2}}}{4} \gamma_{1}^{21}\left(k_{1}\right)=\frac{\omega_{k_{1}} \Delta_{k_{1} B} \omega_{k_{2}} \Delta_{k_{2} B}}{8} \gamma_{0}^{00} .
\end{aligned}
$$

\section{SCHRÖDINGER'S EQUATION}

Let us define the symbol $\Gamma$ by any solution of

$$
\sum_{j=0}^{i}\left(H_{i+2-j}-Q_{\frac{i-j}{2}+1}\right)|0\rangle_{j}=Q_{0}^{-i / 2} \sum_{m n} \int \frac{d^{n} k}{(2 \pi)^{n}} \Gamma_{i}^{m n}\left(k_{1} \cdots k_{n}\right) \phi_{0}^{m} B_{k_{1}}^{\dagger} \cdots B_{k_{n}}^{\dagger}|0\rangle_{0} .
$$

Then the Schrödinger equation

$$
(H-Q)|0\rangle=0
$$

Using

is solved if

$$
\Gamma_{i}^{m n}=0 .
$$

Note that $\Gamma$ is not uniquely defined by (4.1). A necessary and sufficient condition for a solution to Schrödinger's equations is that $\Gamma_{i}^{m n}$ vanishes when summed over all permutations of the $k_{j}$. The number of loops can be defined by counting powers of $\hbar$ and is equal to $i / 2+1$. Note that only integral numbers of loops correct the energy, and so $Q$ vanishes if its subscript is a half-integer. Here $Q$ is defined to be the energy of the ground state. For applications to other states, $Q$ should be replaced with their respective energies.

Let us begin with the one-loop approximation, $i=0$. Using

$$
H_{2}-Q_{1}=\frac{\pi_{0}^{2}}{2}+\int \frac{d k}{2 \pi} \omega_{k} B_{k}^{\dagger} B_{k}
$$

one finds that the Schrödinger equation is satisfied if

$$
\pi_{0}|0\rangle_{0}=B_{k}|0\rangle_{0}=0
$$

These are both satisfied by the initial condition $\gamma_{0}^{m n}=$ $\delta_{m 0} \delta_{n 0}$ of our recursion.

\section{A. Leading corrections}

$$
\begin{aligned}
H_{3}= & \frac{1}{6} \int d x V^{(3)}[\sqrt{\lambda} f(x)]: \phi^{3}(x):_{a}, \\
= & \frac{1}{6} \int d x V^{(3)}[\sqrt{\lambda} f(x)]: \phi^{3}(x):_{b} \\
& +\frac{1}{2} \int d x V^{(3)}[\sqrt{\lambda} f(x)] \phi(x) \mathcal{I}(x),
\end{aligned}
$$

where we have defined $V^{(n)}[\sqrt{\lambda} f(x)]$ to be the $n$th derivative of $\lambda^{-1} V[\sqrt{\lambda} \phi(x)]$ with respect to $\phi(x)$, evaluated at $\phi(x)=f(x)$, one finds that the leading correction to the states (3.10) yields

$\Gamma_{1}^{21}=\sqrt{Q_{0}} \frac{V_{B B k_{1}}}{2}+\frac{\omega_{k_{1}}^{2} \Delta_{k_{1} B}}{2}$,

$\Gamma_{1}^{12}=\sqrt{Q_{0}} \frac{V_{B k_{1} k_{2}}}{2}+\frac{\left(\omega_{k_{1}}-\omega_{k_{2}}\right)\left(\omega_{k_{1}}+\omega_{k_{2}}\right) \Delta_{k_{1} k_{2}}}{2}$,

where we have introduced the notation

$V_{\mathcal{I}^{m} \cdots \mathcal{I}, \alpha_{1} \cdots \alpha_{n}}=\int d x V^{(2 m+n)}[\sqrt{\lambda} f(x)] \mathcal{I}^{m}(x) g_{\alpha_{1}}(x) \cdots g_{\alpha_{n}(x)}$,

At $i=1$ the Schrödinger equation is

$$
H_{3}|0\rangle_{0}+\left(H_{2}-Q_{1}\right)|0\rangle_{1}=0 \text {. }
$$

where $\alpha_{j}$ can be $B$ or $k_{j}$. 
Substituting the identities [12]

$$
\begin{aligned}
V_{B B k} & =\int d x V^{(3)}[\sqrt{\lambda} f(x)] g_{B}(x) \frac{f^{\prime}(x)}{\sqrt{Q_{0}}} g_{k}(x)=\frac{1}{\sqrt{Q_{0}}} \int d x \partial_{x}\left(V^{(2)}[\sqrt{\lambda} f(x)]\right) g_{B}(x) g_{k}(x), \\
& =-\frac{1}{\sqrt{Q_{0}}} \int d x V^{(2)}[\sqrt{\lambda} f(x)]\left(g_{B}^{\prime}(x) g_{k}(x)+g_{B}(x) g_{k}^{\prime}(x)\right), \\
& =-\frac{1}{\sqrt{Q_{0}}} \int d x\left(g_{B}^{\prime}(x) \omega_{k}^{2} g_{k}(x)+g_{B}^{\prime}(x) g_{k}^{\prime \prime}(x)+g_{B}^{\prime \prime}(x) g_{k}^{\prime}(x)\right), \\
& =-\frac{\omega_{k}^{2}}{\sqrt{Q_{0}}} \Delta_{k B}, \\
V_{B k_{1} k_{2}} & =-\frac{1}{\sqrt{Q_{0}}} \int d x V^{(2)}[\sqrt{\lambda} f(x)]\left(g_{k_{1}}^{\prime}(x) g_{k_{2}}(x)+g_{k_{1}}(x) g_{k_{2}}^{\prime}(x)\right), \\
& =-\frac{1}{\sqrt{Q_{0}}} \int d x\left(g_{k_{1}}^{\prime}(x) \omega_{k_{2}}^{2} g_{k_{2}}(x)+g_{k_{1}}^{\prime}(x) g_{k_{2}}^{\prime \prime}(x)+\omega_{k_{1}}^{2} g_{k_{1}}(x) g_{k_{2}}^{\prime}(x)+g_{k_{1}}^{\prime \prime}(x) g_{k_{2}}^{\prime}(x)\right), \\
& =\frac{\omega_{k_{2}}^{2}-\omega_{k_{1}}^{2}}{\sqrt{Q_{0}}} \Delta_{k_{1} k_{2}}
\end{aligned}
$$

into (4.8) one finds $\Gamma=0$, and so these matrix elements of Schrödinger's equation are satisfied by the states (3.10), which were derived from translation invariance alone. This is consistent with our claim that all $\phi_{0}$ descendants $(m>0$ components of states) are determined in terms of $\phi_{0}$ primaries by imposing the eigenvalue of the momentum, in this case zero.

Actually the derivation of the second identity in (4.10) is not quite correct. As the kink is localized by definition, $g_{B}(x)$ tends to zero at large $x$, while $V^{(3)}[\sqrt{\lambda} f(x)]$ tends to a constant. As the remaining terms tend to $e^{-i\left(k_{1}+k_{2}\right) x}$, the integral over $x$ defining the left-hand side converges at large $x$. The problem is that the integration by parts, used to convert $V^{(3)}[\sqrt{\lambda} f(x)] g_{B}$ to $V^{(2)}[\sqrt{\lambda} f(x)]$, leads to a boundary term $V^{(2)}[\sqrt{\lambda} f(x)] g_{k_{1}}(x) g_{k_{2}}(x)$, which does not vanish at large $x$. Instead, it becomes $m e^{-i\left(k_{1}+k_{2}\right) x_{m}}$, where $x_{m}$ is the upper limit of integration. As one takes the limit $x_{m} \rightarrow \infty$, this vanishes by the Riemann-Lebesgue lemma when integrated over any kernel continuous in $k_{1}+k_{2}$. As the left-hand side is finite at $k_{1}+k_{2}=0$ and the dropped boundary term vanishes in the sense of a distribution, the final expression must also vanish in the sense of a distribution. Let us check this for the term

$$
\Delta_{k_{1} k_{2}} \supset i \pi\left(k_{2}-k_{1}\right) \delta\left(k_{1}+k_{2}\right),
$$

which appears for any potential $V$. This term indeed does not contribute to the last line of (4.10) as it vanishes in the sense of a distribution when multiplied by $\omega_{k_{2}}^{2}-\omega_{k_{1}}^{2}$.

Similarly, in our main result (5.9), the $\Delta$ is multiplied by $\left(\omega_{k_{1}}-\omega_{k_{2}}\right)$. The $\delta\left(k_{1}+k_{2}\right)$ term in $\Delta$ therefore does not contribute to the two-loop energy of the ground state.
It does, however, contribute to the two-loop energy of a kink with an excited normal mode [17] where it leads to the recoil kinetic energy of the bulk motion of the kink. We also note that one cannot simply divide (4.10) through by $\left(\omega_{k_{2}}^{2}-\omega_{k_{1}}^{2}\right)$ to obtain $\Delta_{k_{1} k_{2}}$ as a function of $V_{B k_{1} k_{2}}$, as this quantity vanishes on the support of the delta function (4.11).

The other components of the Schrödinger equation at $i=1$ are

$$
\begin{aligned}
& \Gamma_{1}^{01}=\frac{\sqrt{Q_{0}}}{2} V_{\mathcal{I} k_{1}}-\frac{\omega_{k_{1}} \Delta_{k_{1} B}}{2}+\omega_{k_{1}} \gamma_{1}^{01}, \\
& \Gamma_{1}^{03}=\frac{\sqrt{Q_{0}}}{6} V_{k_{1} k_{2} k_{3}}+\left(\omega_{k_{1}}+\omega_{k_{2}}+\omega_{k_{3}}\right) \gamma_{1}^{03}
\end{aligned}
$$

and so the state at order $i=1$ is given by the $\phi_{0}$ descendants in Eqs. (3.10) and (3.12) together with the $\phi_{0}$ primaries

$$
\gamma_{1}^{01}=\frac{\Delta_{k_{1} B}}{2}-\frac{\sqrt{Q_{0}}}{2} Y_{\mathcal{I} k_{1}}, \quad \gamma_{1}^{03}=-\frac{\sqrt{Q_{0}}}{6} Y_{k_{1} k_{2} k_{3}},
$$

where we have defined the reduced potential

$$
Y_{k_{1} \cdots k_{j}}=\frac{V_{k_{1} \cdots k_{j}}}{\omega_{k_{1}}+\cdots+\omega_{k_{j}}}, \quad Y_{\mathcal{I}, k_{1} \cdots k_{j}}=\frac{V_{\mathcal{I}, k_{1} \cdots k_{j}}}{\omega_{k_{1}}+\cdots+\omega_{k_{j}}} .
$$

This is depicted in the top-left panel of Fig. 2. Here one sees that the leading order $\gamma_{0}^{00}$ contribution corresponding to the star contributes to four elements $\gamma_{1}$, shown as squares. Two of these are descendants and so were already fixed by translation invariance. 

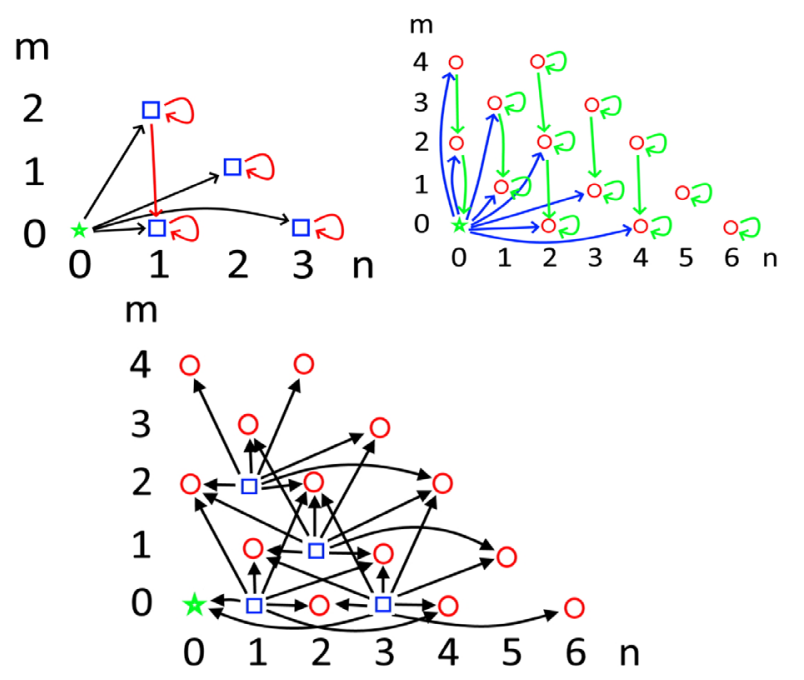

FIG. 2. Terms in the Schrödinger equation: $H_{3}|0\rangle_{0}$ in black and $\left(H_{2}-Q_{1}\right)|0\rangle_{1}$ in red (top left), $\left(H_{4}-Q_{2}\right)|0\rangle_{0}$ in blue and $\left(H_{2}-\right.$ $\left.Q_{1}\right)|0\rangle_{2}$ in green (top right), and $H_{3}|0\rangle_{1}$ in black (bottom).

Note that in models like the $\phi^{4}$ double well, in which the third derivative of the potential is nonzero at the minima, $V_{k_{1} k_{2} k_{3}}$ will have a divergence of the form $\delta\left(\sum_{i} k_{i}\right)$. When integrated over $k$ to determine the state, this divergence leads to finite coefficients. However at two loops it leads to an infrared divergence in the energy of the kink state. As we will see in Sec. V B, this infrared divergence also appears in the vacuum energy and so the kink mass, which is the difference between the energies of the two states, is finite.

\section{B. The kink ground state at two loops}

Translation invariance fixes all $\phi_{0}$-descendant components $\gamma_{i}^{m n}$ in any Hamiltonian eigenstate. The $\phi_{0}$-primary terms $\gamma_{i}^{0 n}$, at each order $i$ are fixed by the Schrödinger equation. Interaction terms relate these coefficients to those at lower orders. Thus the other $\gamma_{i}^{m n}$ are only related to $\gamma_{i}^{0 n}$ by the free Hamiltonian (4.4). More specifically, $\gamma_{i}^{0 n}$ is related to $\gamma_{i}^{2 n}$ by the $\pi_{0}^{2} / 2$ term and to $\gamma_{i}^{0 n}$ by the oscillator term. This allows each $\phi_{0}$ primary $\gamma_{i}^{0 n}$ to be determined from $\gamma_{i}^{2 n}$ and the state at orders less than $i$. In theories, like those considered here, with nonderivative interactions the situation is even simpler because interactions never decrease $m$. Thus Schrödinger's equation determines $\phi_{0}$ primaries $\gamma_{i}^{0 n}$ in terms of $\gamma_{i}^{2 n}$ and $\phi_{0}$ primaries $\gamma_{j}^{0 n^{\prime}}$ with $j<i$. In other words, only the $\phi_{0}$ descendants at $m=2$ are needed. Similarly the energy at each order $i$ is determined by $\gamma_{i}^{20}$ together with the $\phi_{0}$ primaries $\gamma_{j}^{0 n}$ at lower orders $j<i$.

This observation in practice leads to a dramatic reduction in the complexity of calculations of states and energies. For example, to compute the two-loop energy of the kink ground state, one only needs to know $\gamma_{2}^{20}, \gamma_{1}^{01}$, and $\gamma_{1}^{03}$, which themselves are determined from $\gamma_{1}^{21}$ and $\gamma_{1}^{12}$. In this subsection we will complete the calculation of the kink ground state at two loops, corresponding to $i=2(2-1)=2$, by finding the $\phi_{0}$ primaries.

At $i=2$ the Schrödinger equation is

$$
\left(H_{4}-Q_{2}\right)|0\rangle_{0}+H_{3}|0\rangle_{1}+\left(H_{2}-Q_{1}\right)|0\rangle_{2}=0 .
$$

The $H_{4}$ term and $H_{3}$ term are, respectively, shown in the top-right and bottom panels of Fig. 2. In both cases, the goal is to compute the red circles, corresponding to $\gamma_{2}^{m n}$, which lie at the ends of the arrows. Again there is one contribution from each arrow, proportional to the $\gamma$ at the beginning of the arrow.

\section{1. $(m=0, n=6)$}

Let us begin with the simplest element, $\Gamma_{2}^{06}$. The previous argument agrees with Fig. 2 showing that there are two contributions, arising from $\gamma_{1}^{03}$, which was found at the previous order, and from $\gamma_{2}^{06}$ which is to be found now. Defining the total energy

$$
\Omega_{n}=\sum_{j=1}^{n} \omega_{k_{j}}
$$

these contributions are

$H_{3}|0\rangle_{1}^{03} \supset-\frac{1}{36} \int \frac{d^{6} k}{(2 \pi)^{6}} Y_{k_{1} k_{2} k_{3}} V_{k_{4} k_{5} k_{6}} B_{k_{1}}^{\dagger} \cdots B_{k_{6}}^{\dagger}|0\rangle_{0}$,

$H_{2}|0\rangle_{2}^{06}=\frac{1}{Q_{0}} \int \frac{d^{6} k}{(2 \pi)^{6}} \Omega_{6} \gamma_{2}^{06} B_{k_{1}}^{\dagger} \cdots B_{k_{6}}^{\dagger}|0\rangle_{0}$,

and so one finds the matrix element

$$
\gamma_{2}^{06}=\frac{Q_{0}}{36} Y_{k_{1} k_{2} k_{3}} \frac{V_{k_{4} k_{5} k_{6}}}{\Omega_{6}} .
$$

$$
\text { 2. }(m=0, n=4)
$$

To organize the calculations of the other matrix elements, we note that $\Gamma$ may be decomposed into contributions that do not mix with one another. In particular contributions with different numbers of dummy momenta $k^{\prime}$ and with different numbers of powers of the undifferentiated ${ }^{4}$ contraction factor $\mathcal{I}(x)$ together with $V^{(3)}$ do not mix. We will include this decomposition in the subscript of $\Gamma$. Of course each $\Gamma_{i}^{0 n}$ determines $\gamma_{i}^{0 n}$ whose form is not known before $\Gamma_{i}^{0 n}$ is calculated, so terms resulting from $\gamma_{i}^{0 n}$ will not be included in this decomposition.

\footnotetext{
${ }^{4}$ If multiplied by $V^{(4)} g_{B}(x)$ then an integration by parts leads to a differentiated $\mathcal{I}(x)$, which can be evaluated using (2.24) and this argument does not apply. This situation does not arise in the calculation of $\gamma_{2}^{0 n}$ but does arise when verifying that the Schrödinger equation is satisfied in Appendix A.
} 
Let us begin with all contributions $\Gamma_{2 \mathcal{I}}^{04}$ containing a single power of the contraction factor $\mathcal{I}(x)$. These contributions arise from two terms

$$
\begin{aligned}
& H_{3}|0\rangle_{1}^{03} \supset-\frac{1}{12} \int \frac{d^{4} k}{(2 \pi)^{4}} V_{\mathcal{I} k_{4}} Y_{k_{1} k_{2} k_{3}} B_{k_{1}}^{\dagger} \cdots B_{k_{4}}^{\dagger}|0\rangle_{0}, \\
& H_{3}|0\rangle_{1}^{01} \supset-\frac{1}{12} \int \frac{d^{4} k}{(2 \pi)^{4}} Y_{\mathcal{I} k_{4}} V_{k_{1} k_{2} k_{3}} B_{k_{1}}^{\dagger} \cdots B_{k_{4}}^{\dagger}|0\rangle_{0},
\end{aligned}
$$

whose sum yields

$$
\Gamma_{2 \mathcal{I}}^{04}=-\frac{Q_{0}}{12} Y_{\mathcal{I} k_{4}} Y_{k_{1} k_{2} k_{3}} \Omega_{4}
$$

Next let us consider the contributions with one contracted momentum $k^{\prime}$. There is only one

$$
H_{3}|0\rangle_{1}^{03} \supset-\frac{1}{8} \int \frac{d^{4} k}{(2 \pi)^{4}} \int \frac{d k^{\prime}}{2 \pi} Y_{k_{1} k_{2}-k^{\prime}} \frac{V_{k_{3} k_{4}-k^{\prime}}}{\omega_{k^{\prime}}} B_{k_{1}}^{\dagger} \cdots B_{k_{4}}^{\dagger}|0\rangle_{0}
$$

yielding

$$
\Gamma_{2 k^{\prime}}^{04}=-\frac{Q_{0}}{8} \int \frac{d k^{\prime}}{2 \pi} Y_{k_{1} k_{2}-k^{\prime}} \frac{V_{k_{3} k_{4}-k^{\prime}}}{\omega_{k^{\prime}}}
$$

Finally there are three contributions with no $k^{\prime}$

$$
\begin{aligned}
& H_{3}|0\rangle_{1}^{01} \supset \frac{1}{12 \sqrt{Q_{0}}} \int \frac{d^{4} k}{(2 \pi)^{4}} \Delta_{k_{1} B} V_{k_{2} k_{3} k_{4}} B_{k_{1}}^{\dagger} \cdots B_{k_{4}}^{\dagger}|0\rangle_{0}, \\
& \frac{\pi_{0}^{2}}{2}|0\rangle_{2}^{24}=\frac{1}{12 Q_{0}} \int \frac{d^{4} k}{(2 \pi)^{4}}\left[-6 \omega_{k_{1}} \omega_{k_{3}} \Delta_{k_{1} k_{2}} \Delta_{k_{3} k_{4}}+\sqrt{Q_{0}} Y_{k_{1} k_{2} k_{3}} \omega_{k_{4}} \Delta_{k_{4} B}\right] B_{k_{1}}^{\dagger} \cdots B_{k_{4}}^{\dagger}|0\rangle_{0}, \\
& H_{4}|0\rangle_{0}=\frac{1}{24} \int \frac{d^{4} k}{(2 \pi)^{4}} V_{k_{1} k_{2} k_{3} k_{4}} B_{k_{1}}^{\dagger} \cdots B_{k_{4}}^{\dagger}|0\rangle_{0},
\end{aligned}
$$

which sum to

$$
\Gamma_{2 k^{\prime 0}}^{04}=\frac{\sqrt{Q_{0}}}{12} Y_{k_{1} k_{2} k_{3}} \Delta_{k_{4} B} \Omega_{4}-\frac{\omega_{k_{1}} \omega_{k_{3}}}{2} \Delta_{k_{1} k_{2}} \Delta_{k_{3} k_{4}}+\frac{Q_{0}}{24} V_{k_{1} k_{2} k_{3} k_{4}}
$$

The final contribution to $\Gamma_{2}^{04}$ arises from

$$
\int \frac{d k}{2 \pi} \omega_{k} B_{k}^{\dagger} B_{-k}|0\rangle_{2}^{04}=\frac{1}{Q_{0}} \int \frac{d^{4} k}{(2 \pi)^{4}} \Omega_{4} \gamma_{2}^{04} B_{k_{1}}^{\dagger} \cdots B_{k_{4}}^{\dagger}|0\rangle_{0}
$$

and is

$$
\Gamma_{2 f}^{04}=\Omega_{4} \gamma_{2}^{04}
$$

The Schrödinger equation

$$
0=\Gamma_{2}^{04}=\Gamma_{2 f}^{04}+\Gamma_{2 \mathcal{I}}^{04}+\Gamma_{2 k^{\prime 0}}^{04}+\Gamma_{2 k^{\prime}}^{04}
$$

then yields the matrix element 


$$
\begin{aligned}
\gamma_{2}^{04} & =-\frac{\Gamma_{2 \mathcal{I}}^{04}+\Gamma_{2 k^{\prime}}^{04}+\Gamma_{2 k^{\prime 0}}^{04}}{\Omega_{4}}, \\
& =\frac{Q_{0}}{12} Y_{\mathcal{I} k_{4}} Y_{k_{1} k_{2} k_{3}}-\frac{\sqrt{Q_{0}}}{12} Y_{k_{1} k_{2} k_{3}} \Delta_{k_{4} B}+\frac{\omega_{k_{1}} \omega_{k_{3}}}{2 \Omega_{4}} \Delta_{k_{1} k_{2}} \Delta_{k_{3} k_{4}}-\frac{Q_{0}}{24} Y_{k_{1} k_{2} k_{3} k_{4}}+\frac{Q_{0}}{8 \Omega_{4}} \int \frac{d k^{\prime}}{2 \pi} Y_{k_{1} k_{2}-k^{\prime}} \frac{V_{k_{3} k_{4}-k^{\prime}}}{\omega_{k^{\prime}}} .
\end{aligned}
$$

Note that in models like the Sine-Gordon model, in which the fourth derivative of the potential is nonzero at the minima, $Y_{k_{1} k_{2} k_{3} k_{4}}$ will have a divergence of the form $\delta\left(\sum_{i} k_{i}\right)$. When integrated over $k$ to determine the state, this divergence leads to finite coefficients. However at three loops it leads to an infrared divergence in the energy of the kink state. As in the twoloop divergence in the $\phi^{4}$ kink energy, this divergence also appears in the vacuum energy and so the kink mass remains finite. We expect such cancellations at all loops, as the infrared divergences arise from a regime in $x$ where $f(x)$ is equal to a vacuum value, and so the energy contribution from the kink and vacuum sector should agree.

\section{3. $(m=0, n=2)$}

The last matrix element needed to fix the ground state at two loops is $(m=0, n=2)$. There is one contribution with two powers of the contraction factor $\mathcal{I}$

$$
H_{3}|0\rangle_{1}^{01} \supset-\frac{1}{4} \int \frac{d^{2} k}{(2 \pi)^{2}} Y_{\mathcal{I} k_{1}} V_{\mathcal{I} k_{2}} B_{k_{1}}^{\dagger} B_{k_{2}}^{\dagger}|0\rangle_{0},
$$

which, after adding an antisymmetric term which does not affect the sum, yields

$$
\Gamma_{2 \mathcal{I}^{2}}^{02}=-\frac{Q_{0}}{8} Y_{\mathcal{I} k_{1}} Y_{\mathcal{I} k_{2}} \Omega_{2}
$$

There are four contributions with a single power of $\mathcal{I}$

$$
\begin{aligned}
& \frac{\pi_{0}^{2}}{2}|0\rangle_{2}^{22} \supset \frac{1}{4 \sqrt{Q_{0}}} \int \frac{d^{2} k}{(2 \pi)^{2}} Y_{\mathcal{I} k_{1}} \omega_{k_{2}} \Delta_{k_{2} B} B_{k_{1}}^{\dagger} B_{k_{2}}^{\dagger}|0\rangle_{0}, \\
& H_{3}|0\rangle_{1}^{01} \supset \int \frac{d^{2} k}{(2 \pi)^{2}}\left(\frac{1}{4 \sqrt{Q_{0}}} V_{\mathcal{I} k_{2}} \Delta_{k_{1} B}-\frac{1}{8} \int \frac{d k^{\prime}}{2 \pi} Y_{\mathcal{I} k^{\prime}} \frac{V_{k_{1} k_{2}-k^{\prime}}}{\omega_{k^{\prime}}}\right) B_{k_{1}}^{\dagger} B_{k_{2}}^{\dagger}|0\rangle_{0}, \\
& H_{4}|0\rangle_{0} \supset \frac{1}{4} \int \frac{d^{2} k}{(2 \pi)^{2}} V_{\mathcal{I} k_{1} k_{2}} B_{k_{1}}^{\dagger} B_{k_{2}}^{\dagger}|0\rangle_{0}, \\
& H_{3}|0\rangle_{1}^{03} \supset-\frac{1}{8} \int \frac{d^{2} k}{(2 \pi)^{2}} \int \frac{d k^{\prime}}{2 \pi} Y_{\mathcal{I} k^{\prime}} Y_{k_{1} k_{2}-k^{\prime}} B_{k_{1}}^{\dagger} B_{k_{2}}^{\dagger}|0\rangle_{0},
\end{aligned}
$$

which together contribute

$$
\Gamma_{2 \mathcal{I}}^{02}=\frac{\sqrt{Q_{0}}}{4} Y_{\mathcal{I} k_{1}} \Delta_{k_{2} B} \Omega_{2}+\frac{Q_{0} V_{\mathcal{I} k_{1} k_{2}}}{4}-\frac{Q_{0}}{8} \int \frac{d k^{\prime}}{2 \pi} Y_{\mathcal{I} k^{\prime}} Y_{k_{1} k_{2}-k^{\prime}}\left(2+\frac{\Omega_{2}}{\omega_{k^{\prime}}}\right)
$$

Now we will organize the terms with no powers of $\mathcal{I}$ by the number of contracted momenta $k^{\prime}$. There is one term with two contracted momenta

$$
H_{3}|0\rangle_{1}^{03} \supset-\frac{1}{8} \int \frac{d^{2} k}{(2 \pi)^{2}} \int \frac{d^{2} k^{\prime}}{(2 \pi)^{2}} Y_{k_{1} k_{1}^{\prime} k_{2}^{\prime}} \frac{V_{k_{2}-k_{1}^{\prime}-k_{2}^{\prime}}}{\omega_{k_{1}^{\prime}} \omega_{k_{2}^{\prime}}} B_{k_{1}}^{\dagger} B_{k_{2}}^{\dagger}|0\rangle_{0},
$$

yielding

$$
\Gamma_{2 k^{2}}^{02}=-\frac{Q_{0}}{8} \int \frac{d^{2} k^{\prime}}{(2 \pi)^{2}} Y_{k_{1} k_{1}^{\prime} k_{2}^{\prime}} \frac{V_{k_{2}-k_{1}^{\prime}-k_{2}^{\prime}}}{\omega_{k_{1}^{\prime}} \omega_{k_{2}^{\prime}}}
$$

There are two sources of terms with no $\mathcal{I}$ and a single $k^{\prime}$ 


$$
\begin{aligned}
& H_{3}|0\rangle_{1}^{01} \supset \frac{1}{8 \sqrt{Q_{0}}} \int \frac{d^{2} k}{(2 \pi)^{2}} \int \frac{d k^{\prime}}{2 \pi} \frac{V_{k_{1} k_{2} k^{\prime}}}{\omega_{k^{\prime}}} \Delta_{-k^{\prime} B} B_{k_{1}}^{\dagger} B_{k_{2}}^{\dagger}|0\rangle_{0}, \\
& \frac{\pi_{0}^{2}}{2}|0\rangle_{2}^{22} \supset \int \frac{d^{2} k}{(2 \pi)^{2}} \int \frac{d k^{\prime}}{2 \pi}\left[-\frac{1}{8 \sqrt{Q_{0}}} Y_{k_{1} k_{2} k^{\prime}} \Delta_{-k^{\prime} B}+\frac{1}{4 Q_{0}} \Delta_{k_{1} k^{\prime}} \Delta_{-k^{\prime} k_{2}}\left(1+\frac{\omega_{k_{2}}}{\omega_{k^{\prime}}}\right)\left(\omega_{k_{1}}-\omega_{k^{\prime}}\right)\right] B_{k_{1}}^{\dagger} B_{k_{2}}^{\dagger}|0\rangle_{0},
\end{aligned}
$$

which contribute

$$
\Gamma_{2 k^{\prime}}^{02}=\int \frac{d k^{\prime}}{2 \pi}\left[\frac{\sqrt{Q_{0}}}{8} \frac{\Omega_{2}}{\omega_{k^{\prime}}} Y_{k_{1} k_{2} k^{\prime}} \Delta_{-k^{\prime} B}+\frac{1}{4} \Delta_{k_{1} k^{\prime}} \Delta_{-k^{\prime} k_{2}}\left(\frac{\omega_{k_{1}} \omega_{k_{2}}}{\omega_{k^{\prime}}}-\omega_{k^{\prime}}\right)\right]
$$

Finally the terms with neither $\mathcal{I}$ nor $k^{\prime}$ are

$$
\frac{\pi_{0}^{2}}{2}|0\rangle_{2}^{22} \supset-\frac{3}{8 Q_{0}} \int \frac{d^{2} k}{(2 \pi)^{2}} \Omega_{2} \Delta_{k_{1} B} \Delta_{k_{2} B} B_{k_{1}}^{\dagger} B_{k_{2}}^{\dagger}|0\rangle_{0},
$$

and so

$$
\Gamma_{2 k^{\prime 0}}^{02}=-\frac{3}{8} \Omega_{2} \Delta_{k_{1} B} \Delta_{k_{2} B}
$$

As in the previous cases,

$$
\Gamma_{2 f}^{02}=\Omega_{2} \gamma_{2}^{02}
$$

and so the Schrödinger equation

$$
0=\Gamma_{2}^{02}=\Gamma_{2 f}^{02}+\Gamma_{2 \mathcal{I}^{2}}^{02}+\Gamma_{2 \mathcal{I}}^{02}+\Gamma_{2 k^{\prime 2}}^{02}+\Gamma_{2 k^{\prime}}^{02}+\Gamma_{2 k^{0}}^{02}
$$

fixes the last matrix element

$$
\begin{aligned}
\gamma_{2}^{02}= & -\frac{\Gamma_{2 \mathcal{I}^{2}}^{02}+\Gamma_{2 \mathcal{I}}^{02}+\Gamma_{2 k^{\prime 2}}^{02}+\Gamma_{2 k^{\prime}}^{02}+\Gamma_{2 k^{\prime 0}}^{02}}{\Omega_{2}}, \\
= & \frac{Q_{0}}{8} Y_{\mathcal{I} k_{1}} Y_{\mathcal{I} k_{2}}-\frac{\sqrt{Q_{0}}}{4} Y_{\mathcal{I} k_{1}} \Delta_{k_{2} B}-\frac{Q_{0} V_{\mathcal{I} k_{1} k_{2}}}{4 \Omega_{2}}+\frac{3}{8} \Delta_{k_{1} B} \Delta_{k_{2} B}+\frac{Q_{0}}{8 \Omega_{2}} \int \frac{d^{2} k^{\prime}}{(2 \pi)^{2}} Y_{k_{1} k_{1}^{\prime} k_{2}^{\prime}} \frac{V_{k_{2}-k_{1}^{\prime}-k_{2}^{\prime}}}{\omega_{k_{1}^{\prime}} \omega_{k_{2}^{\prime}}} \\
& +\int \frac{d k^{\prime}}{2 \pi}\left[-\frac{\sqrt{Q_{0}}}{8} \frac{1}{\omega_{k^{\prime}}} Y_{k_{1} k_{2} k^{\prime}} \Delta_{-k^{\prime} B}+\frac{1}{4 \Omega_{2}}\left(Q_{0} Y_{\mathcal{I} k^{\prime}} Y_{k_{1} k_{2}-k^{\prime}}\left(1+\frac{\Omega_{2}}{2 \omega_{k^{\prime}}}\right)+\Delta_{k_{1} k^{\prime}} \Delta_{-k^{\prime} k_{2}}\left(\omega_{k^{\prime}}-\frac{\omega_{k_{1}} \omega_{k_{2}}}{\omega_{k^{\prime}}}\right)\right)\right] .
\end{aligned}
$$

\section{THE KINK MASS}

\section{A. The energy of the kink ground state}

The last Schrödinger equation is $\Gamma_{2}^{00}=0$. This does not fix $\gamma_{2}^{00}$ because $\Gamma_{2}^{00}$ does not depend on $\gamma_{2}^{00}$. This is reasonable because any value of $\gamma_{2}^{00}$ can be absorbed into the normalization of the state. Thus one may normalize the ground state so that

$$
\gamma_{i}^{00}=\delta_{i 0} .
$$


Let us now solve this last Schrödinger equation. There are two terms with two powers of the contraction factor $\mathcal{I}$

$$
\begin{aligned}
& H_{4}|0\rangle_{0} \supset \frac{V_{\mathcal{I I}}}{8}|0\rangle_{0}, \\
& H_{3}|0\rangle_{1}^{01} \supset-\frac{1}{8} \int \frac{d k^{\prime}}{2 \pi} Y_{\mathcal{I} k^{\prime}} Y_{\mathcal{I}-k^{\prime}}|0\rangle_{0},
\end{aligned}
$$

yielding

$$
\Gamma_{2 \mathcal{I}^{2}}^{00} \supset \frac{Q_{0}}{8}\left(V_{\mathcal{I I}}-\int \frac{d k^{\prime}}{2 \pi} Y_{\mathcal{I} k^{\prime}} Y_{\mathcal{I}-k^{\prime}}\right)
$$

There are also two terms with a single factor of $\mathcal{I}$

$$
\begin{aligned}
& H_{3}|0\rangle_{1}^{01} \supset \frac{1}{8 \sqrt{Q_{0}}} \int \frac{d k^{\prime}}{2 \pi} Y_{\mathcal{I} k^{\prime}} \Delta_{-k^{\prime} B}|0\rangle_{0}, \\
& \frac{\pi_{0}^{2}}{2}|0\rangle_{2}^{20} \supset-\frac{1}{8 \sqrt{Q_{0}}} \int \frac{d k^{\prime}}{2 \pi} Y_{\mathcal{I} k^{\prime}} \Delta_{-k^{\prime} B}|0\rangle_{0},
\end{aligned}
$$

which precisely cancel. The terms with no factors of $\mathcal{I}$ can be organized by the number of contracted momenta $k^{\prime}$. There is one term with 3,2 , and 1 momenta, respectively, which for brevity we summarize together

$$
\begin{aligned}
& H_{3}|0\rangle_{1}^{03} \supset-\frac{1}{48} \int \frac{d^{3} k^{\prime}}{(2 \pi)^{3}} Y_{k_{1}^{\prime} k_{2}^{\prime} k_{3}^{\prime}} \frac{V_{-k_{1}^{\prime}-k_{2}^{\prime}-k_{3}^{\prime}}}{\omega_{k_{1}^{\prime}} \omega_{k_{2}^{\prime}} \omega_{k_{3}^{\prime}}}, \\
& \frac{\pi_{0}^{2}}{2}|0\rangle_{2}^{20} \supset \frac{1}{16 Q_{0}} \int \frac{d^{2} k^{\prime}}{(2 \pi)^{2}} \frac{\left(\omega_{k_{1}^{\prime}}-\omega_{k_{2}^{\prime}}\right)^{2}}{\omega_{k_{1}^{\prime}} \omega_{k_{2}^{\prime}}} \Delta_{k_{1}^{\prime} k_{2}^{\prime}} \Delta_{-k_{1}^{\prime}-k_{2}^{\prime}}, \\
& \frac{\pi_{0}^{2}}{2}|0\rangle_{2}^{20} \supset-\frac{1}{8 Q_{0}} \int \frac{d k^{\prime}}{2 \pi} \Delta_{B k^{\prime}} \Delta_{B-k^{\prime}} .
\end{aligned}
$$

As

$$
g_{k}^{*}(x)=g_{-k}(x)
$$

the symbols $\Delta, V$, and $Y$ are all complex conjugated when all of their $k$ arguments are negated. Therefore these contributions can each be rewritten as norms squared and so are real. The corresponding $\Gamma$ can therefore be written

$$
\begin{aligned}
\Gamma_{2 k^{\prime 3}}^{00} & =-\frac{Q_{0}}{48} \int \frac{d^{3} k^{\prime}}{(2 \pi)^{3}} \frac{\left|V_{k_{1}^{\prime} k_{2}^{\prime} k_{3}^{\prime}}\right|^{2} \omega_{k_{1}^{\prime}} \omega_{k_{2}^{\prime}} \omega_{k_{3}^{\prime}}\left(\omega_{k_{1}^{\prime}}+\omega_{k_{2}^{\prime}}+\omega_{k_{3}^{\prime}}\right)}{\Gamma_{2 k^{\prime 2}}^{00}}=\frac{1}{16} \int \frac{d^{2} k^{\prime}}{(2 \pi)^{2}} \frac{\left|\left(\omega_{k_{1}^{\prime}}-\omega_{k_{2}^{\prime}}\right) \Delta_{k_{1}^{\prime} k_{2}^{\prime}}\right|^{2}}{\omega_{k_{1}^{\prime}} \omega_{k_{2}^{\prime}}}, \\
\Gamma_{2 k^{\prime}}^{00} & =-\frac{1}{8} \int \frac{d k^{\prime}}{2 \pi}\left|\Delta_{k^{\prime} B}\right|^{2} .
\end{aligned}
$$

The last term may be written in a more convenient form using the completeness relation (2.16)

$$
\begin{aligned}
\int \frac{d k}{2 \pi} \Delta_{k B} \Delta_{-k B} & =\frac{1}{Q_{0}} \int d x \int d y \int \frac{d k}{2 \pi} g_{k}(x) g_{-k}(y) f^{\prime \prime}(x) f^{\prime \prime}(y), \\
& =\frac{1}{Q_{0}} \int d x \int d y\left(\delta(x-y)-g_{B}(x) g_{B}(y)\right) f^{\prime \prime}(x) f^{\prime \prime}(y)=\frac{1}{Q_{0}} \int d x\left|f^{\prime \prime}(x)\right|^{2},
\end{aligned}
$$

where the $g_{B}(x) f^{\prime \prime}(x)$ integrals vanish because they are proportional to the total derivative of $g_{B}^{2}(x)$.

The Schrödinger equation then gives the two-loop energy

$$
\begin{aligned}
Q_{2}= & \frac{1}{Q_{0}}\left(\Gamma_{2 \mathcal{I}^{2}}^{00}+\Gamma_{2 k^{\prime 3}}^{00}+\Gamma_{2 k^{\prime 2}}^{00}+\Gamma_{2 k^{\prime}}^{00}\right), \\
= & \frac{V_{\mathcal{I I}}}{8}-\frac{1}{8} \int \frac{d k^{\prime}}{2 \pi}\left|Y_{\mathcal{I} k^{\prime}}\right|^{2}-\frac{1}{48} \int \frac{d^{3} k^{\prime}}{(2 \pi)^{3}} \frac{\left|V_{k_{1}^{\prime} k_{2}^{\prime} k_{3}^{\prime}}\right|^{2}}{\omega_{k_{1}^{\prime}} \omega_{k_{2}^{\prime}} \omega_{k_{3}^{\prime}}\left(\omega_{k_{1}^{\prime}}+\omega_{k_{2}^{\prime}}+\omega_{k_{3}^{\prime}}\right)} \\
& +\frac{1}{16 Q_{0}} \int \frac{d^{2} k^{\prime}}{(2 \pi)^{2}} \frac{\left|\left(\omega_{k_{1}^{\prime}}-\omega_{k_{2}^{\prime}}\right) \Delta_{k_{1}^{\prime} k_{2}^{\prime}}\right|^{2}}{\omega_{k_{1}^{\prime}} \omega_{k_{2}^{\prime}}}-\frac{1}{8 Q_{0}^{2}} \int d x\left|f^{\prime \prime}(x)\right|^{2} .
\end{aligned}
$$

To our knowledge, this is the first time that the two-loop energy has been calculated for kinks that need be neither integrable nor supersymmetric. The explicit calculation of Refs. [18,19], in the case of the Sine-Gordon model, did not require integrability and so could be repeated in this general setting. However in that case we stress that the energy was found by summing 13 divergent Feynman diagrams, and carefully regulating and subtracting the divergences. Here 
instead we find five terms, each of which is already UV finite. Let us identify each of these terms.

When changing from plane wave to normal mode normal ordering, so that $\mathrm{H}_{2}$ annihilates the one-loop kink ground state, the interaction Hamiltonian acquired constant and tadpole terms. The first two terms in (5.9) are just the corresponding leading shifts to the energy, equal to the constant $V_{\mathcal{I I}}$ plus the first perturbative contribution from the tadpole $V_{\mathcal{I} k^{\prime}}$. The next term is the usual one-loop perturbation theory correction to an energy arising from a cubic interaction, and is given by the same expression as in the vacuum sector (5.16) with plane waves replaced by normal modes. The fourth term is a correction to the third term arising from the fact that derivative operators mix the normal modes, which is not the case for plane waves. The last term was found long ago $[18,20]$ using the collective coordinate approach, where it appeared as the leading term in an expansion of the denominator of an effective Hamiltonian, which came from a canonical transformation that separated a nonlinear extension of $\phi_{B}(x)$. The manipulations that led to its appearance here are very different from those in the collective coordinate approach, but it is reassuring to see this agreement in the result.

The only trace of renormalization can be found in the first two terms, in the function $\mathcal{I}(x)$ which is the expected difference between two divergent sums weighted by $1 / \omega_{k}$ and $1 / \omega_{p}$, respectively. In models such as the $\phi^{4}$ double well, in which the potential has a nonvanishing third derivative at the minima, the third term will be IR divergent. This divergence arises from the region far from the kink, and so its contribution to the kink mass will be canceled by the same IR divergence in the vacuum energy, which we will now calculate.

\section{B. Vacuum sector energy}

The kink mass is generally not $Q_{2}$. It is $Q_{2}-E_{1}$ where $E_{1}$ is the one-loop correction to the vacuum sector energy, as this contributes at the same order. It is easily computed in perturbation theory. Decompose the field in terms of plane waves as

$$
\phi(x)=\int \frac{d p}{2 \pi}\left(A_{p}^{\dagger}+\frac{A_{-p}}{2 \omega_{p}}\right) e^{-i p x}
$$

and the free and interaction Hamiltonians can be written

$$
\begin{aligned}
H_{2} & =\int \frac{d p}{2 \pi} \omega_{p} A_{p}^{\dagger} A_{p}, \\
H_{n>2} & =\frac{1}{n !} \int d x V^{(n)}\left[\phi_{0}\right]: \phi^{n}(x):_{a},
\end{aligned}
$$

where $\phi_{0}$ is the minimum of $V$ corresponding to the vacuum. Then the first order of perturbation theory

$$
H_{3}|\Omega\rangle_{0}+H_{2}|\Omega\rangle_{1}=0
$$

yields the first order correction $|\Omega\rangle_{1}$ to the vacuum state $|\Omega\rangle$

$$
\begin{aligned}
|\Omega\rangle_{1}= & -\frac{V^{(3)}\left[\phi_{0}\right]}{6} \int \frac{d^{3} p}{(2 \pi)^{3}} \frac{2 \pi \delta\left(p_{1}+p_{2}+p_{3}\right)}{\omega_{p_{1}}+\omega_{p_{2}}+\omega_{p_{3}}} \\
& \times A_{p_{1}}^{\dagger} A_{p_{2}}^{\dagger} A_{p_{3}}^{\dagger}|\Omega\rangle_{0} .
\end{aligned}
$$

Acting again with $H_{3}$, the $|\Omega\rangle_{0}$ term yields the one loop correction to the energy

$$
\begin{aligned}
H_{3}|\Omega\rangle_{1} & \supset-\frac{\left(V^{(3)}\left[\phi_{0}\right]\right)^{2}}{48} \int d x \int \frac{d^{3} p^{\prime}}{(2 \pi)^{3}} e^{-i x\left(p_{1}^{\prime}+p_{2}^{\prime}+p_{3}^{\prime}\right)} \frac{2 \pi \delta\left(p_{1}+p_{2}+p_{3}\right)}{\omega_{p_{1}^{\prime}} \omega_{p_{2}^{\prime}} \omega_{p_{3}^{\prime}}\left(\omega_{p_{1}^{\prime}}+\omega_{p_{2}^{\prime}}+\omega_{p_{3}^{\prime}}\right)}|\Omega\rangle_{0}, \\
& =-\frac{\left(V^{(3)}\left[\phi_{0}\right]\right)^{2}}{48} L \int \frac{d^{3} p^{\prime}}{(2 \pi)^{3}} \frac{2 \pi \delta\left(p_{1}^{\prime}+p_{2}^{\prime}+p_{3}^{\prime}\right)}{\omega_{p_{1}^{\prime}} \omega_{p_{2}^{\prime}} \omega_{p_{3}^{\prime}}\left(\omega_{p_{1}^{\prime}}+\omega_{p_{2}^{\prime}}+\omega_{p_{3}^{\prime}}\right)}|\Omega\rangle_{0},
\end{aligned}
$$

where $L$ is the length of the spatial direction, ${ }^{5}$ which serves as an infrared cutoff.

The subleading correction to the Schrödinger equation is

$$
\left(H_{4}-E_{1}\right)|\Omega\rangle_{0}+H_{3}|\Omega\rangle_{1}+H_{2}|\Omega\rangle_{2}=0 .
$$

\footnotetext{
${ }^{5}$ Here we are cavalier with boundary conditions, as the theory contains only scalar fields. In practice, we simply subtract the kink and vacuum energy densities before performing the $x$ integration, in which case the integral converges. In a theory with fermions a more careful approach may be warranted, for example adding a distant antikink to each kink to allow identical boundary conditions in each sector.
}

As $H_{4}$ is normal ordered, $H_{4}|\Omega\rangle_{0}$ is orthogonal to $|\Omega\rangle_{0}$ and so does not contribute to $E_{1}$. We will chose $|\Omega\rangle_{2}$ to be orthogonal to $|\Omega\rangle_{0}$ so that the last term does not contribute to $E_{1}$. Then $E_{1}$ can be read off of (5.14). Evaluating the delta function, this is

$$
\begin{aligned}
E_{1}= & -\frac{\left(V^{(3)}\left[\phi_{0}\right]\right)^{2}}{48} \\
& \times L \int \frac{d^{2} p^{\prime}}{(2 \pi)^{2}} \frac{1}{\omega_{p_{1}^{\prime}} \omega_{p_{2}^{\prime}} \omega_{p_{1}^{\prime}+p_{2}^{\prime}}\left(\omega_{p_{1}^{\prime}}+\omega_{p_{2}^{\prime}}+\omega_{p_{1}^{\prime}+p_{2}^{\prime}}\right)} .
\end{aligned}
$$


The dependence on the infrared cutoff $L$ implies that we have calculated an energy density, and not an energy. When this energy density is nonvanishing, it must be subtracted from the kink ground state energy to obtain the kink mass. The kink mass will be finite only if these divergences cancel. This procedure depends on the matching of the infrared divergences, which can be achieved for example if the energy densities are subtracted before they are integrated. If the potential is symmetric about the minimum $\phi_{0}$, as it is in the case of the SineGordon model but not the $\phi^{4}$ double well, $V^{(3)}\left[\phi_{0}\right]$ vanishes, and so $E_{1}=0$ and this complication is avoided.
This procedure is performed in the case of the $\phi^{4}$ model in Ref. [21].

\section{The Sine-Gordon model}

In the case of the Sine-Gordon model, the two-loop mass has been conjectured in [22] and calculated in [5,18,19,23]. It is of course dependent upon the renormalization scheme [22] although in some schemes there is a renormalization group flow invariant coupling that provides a universal relation between the kink and meson mass. No such relation may be expected to hold in general as there are other schemes in which the coupling may be shifted by any finite amount at any scale.

Using the well-known Sine-Gordon normal modes [12]

$$
g_{k}(x)=\frac{e^{-i k x} \operatorname{sign}(k)}{\omega_{k}}(k-i m \tanh (m x)), \quad g_{B}(x)=\sqrt{\frac{m}{2}} \operatorname{sech}(m x),
$$

a contour integration yields

$$
\begin{aligned}
\Delta_{k B} & =\frac{i \pi \omega_{k}}{\sqrt{8 M}} \operatorname{sech}\left(\frac{k \pi}{2 M}\right) \operatorname{sign}(k), \\
\Delta_{k_{1} k_{2}} & =-i\left(k_{1}-k_{2}\right) \pi \delta\left(k_{1}+k_{2}\right)+\frac{i \pi}{2} \frac{\left(k_{2}^{2}-k_{1}^{2}\right)}{\omega_{k_{1}} \omega_{k_{2}}} \operatorname{csch}\left(\frac{\pi\left(k_{1}+k_{2}\right)}{2 M}\right) \operatorname{sign}\left(k_{1} k_{2}\right) .
\end{aligned}
$$

Using

$$
V^{(3)}[\sqrt{\lambda} f(x)]=2 M^{2} g \tanh (M x) \operatorname{sech}(M x)
$$

we obtain

$V_{k_{1} k_{2} k_{3}}=\frac{\pi i}{4} g \operatorname{sign}\left(k_{1} k_{2} k_{3}\right) \operatorname{sech}\left(\frac{\pi\left(k_{1}+k_{2}+k_{3}\right)}{2 M}\right)\left[-\left(\frac{\omega_{k_{1}}^{3}}{\omega_{k_{2}} \omega_{k_{3}}}+\frac{\omega_{k_{2}}^{3}}{\omega_{k_{1}} \omega_{k_{3}}}+\frac{\omega_{k_{3}}^{3}}{\omega_{k_{1}} \omega_{k_{2}}}\right)+2\left(\frac{\omega_{k_{1}} \omega_{k_{2}}}{\omega_{k_{3}}}+\frac{\omega_{k_{1}} \omega_{k_{3}}}{\omega_{k_{2}}}+\frac{\omega_{k_{2}} \omega_{k_{3}}}{\omega_{k_{1}}}\right)\right]$.

We have evaluated the energy (5.9) term by term.

In the first two terms, $\mathcal{I}(x)$ appears. It was calculated in Ref. [12] by integrating the general identity (2.24). Using the present conventions

$$
\mathcal{I}(x)=-\frac{\operatorname{sech}^{2}(M x)}{2 \pi}
$$

and so

$$
V_{\mathcal{I} k}=\frac{i}{8 M^{2}} g \omega_{k}^{3} \operatorname{sign}(k) \operatorname{sech}\left(\frac{\pi k}{2 M}\right) .
$$

Using one finds $M \lambda /\left(40 \pi^{2}\right)$ and $-M \lambda /\left(120 \pi^{2}\right)$ for the first and second terms of (5.9). In the fourth term, the delta function in (5.18) is multiplied by a zero, which leads to a vanishing contribution, as can be checked directly by considering the case $k_{1}=k_{2}$ separately from the beginning. Fixing the mass $M$ and coupling $\sqrt{\lambda}$ to unity, the third, fourth, and fifth terms are equal to terms which may be found in Ref. [18] and they were evaluated analytically by Verwaest who found that the sum of the third and fourth is $-M \lambda /\left(60 \pi^{2}\right)$ while the fifth is $-M \lambda / 192$. Altogether we find that the two-loop correction to the kink mass is 


$$
Q_{2}=-\frac{M \lambda}{192}
$$

in agreement with the literature. As shown in Appendix B our normal ordering prescription yields the same meson mass as Ref. [18] and so the soliton to meson mass ratio agrees, in accordance with Refs. [22,23].

\section{REMARKS}

Calculations of masses of quantum kinks have been an industry from Ref. [1] to Refs. [24,25]. So far these calculations have been largely at one loop, where they are described by a free theory, with the exception of integrable and supersymmetric models. In this paper, we have calculated the two-loop masses of scalar kinks in theories with arbitrary potentials. We have also explicitly constructed their states, with the $\phi_{0}$ descendants calculated in Sec. III using translation invariance and the $\phi_{0}$-primary components in Sec. IV using the Schrödinger equation. These constructions we feel are even more interesting than the masses, as they allow one to compute matrix elements and so open the door to understanding the phenomenology [26], such as scattering [27-29] and acceleration [30,31] of quantum kinks beyond the harmonic oscillator approximation. For example, one may calculate form factors $[32,33]$.

While we only calculated the ground state, starting our recursion with a superposition of normal modes would have allowed us to apply the same strategy to an arbitrary state in the one-kink sector.

The key step in our calculation was perturbatively imposing the translation invariance conditions, which fixed most matrix elements of the state, the $\phi_{0}$ descendants, in terms of a few coefficients, the $\phi_{0}$ primaries. The $\phi_{0^{-}}$ primary components needed to be fixed using ordinary perturbation theory. More generally, in the case of any translation-invariant Hamiltonian, as the Hamiltonian and momentum operators commute, a basis of all Hamiltonian eigenstates may be obtained by first fixing the momentum to obtain the $\phi_{0}$ descendant matrix elements in terms of $\phi_{0^{-}}$ primary matrix elements $\gamma_{i}^{0 n}$ and then using the Schrödinger equation to fix the $\phi_{0}$-primary matrix elements.

In the case of a BPS ${ }^{6}$ state in a supersymmetric model one may first impose both translation invariance and also that the state be invariant under the preserved supersymmetries. Presumably this will strongly constrain the state. The big question is whether, in a sufficiently supersymmetric model, this may constrain the state sufficiently that perturbation theory is no longer required. In this case, one would have finally opened the door to a truly quantum understanding of nonperturbative solitons. More precisely, one could understand the physical mechanisms at work behind the nonrenormalization theorems. This of course is a prerequisite for applying lessons from supersymmetric theories to Yang-Mills.

\section{ACKNOWLEDGMENTS}

J. E. is supported by the CAS Key Research Program of Frontier Sciences Grant No. QYZDY-SSW-SLH006 and the NSFC MianShang Grants No. 11875296 and No. 11675223. J.E. also thanks the Recruitment Program of High-end Foreign Experts for support.

\section{APPENDIX A: CHECKING SCHRÖDINGER'S EQUATION}

We have derived the two-loop ground state using translation invariance together with Schrödinger's equation. We restricted Schrödinger's equation to the $\phi_{0}$ primaries, the subspace of the Fock space with no $\phi_{0}$ acting on $|0\rangle_{0}$, but we argued that, since the Hamiltonian and momentum operators commute, we expect our solutions to solve the Schrödinger equation in the full Fock space. By imposing a condition on the momentum it is not possible that we lose the ground state solution, since it indeed must have zero momentum. Furthermore, since the solution that we find, given the one-loop contribution, is unique, it must be the ground state.

In this appendix we explicitly check this claim by inserting our two-loop state into the Schrödinger equation and showing that it vanishes on the full Fock space. More precisely, we compute the various $\phi_{0}$-descendant components $\Gamma_{2}^{m n}$ at $m>0$ and show that they each vanish as claimed. Recall that in Sec. IV B we found the $\phi_{0}$ primaries $\gamma_{2}^{0 n}$ by imposing that $\Gamma_{2}^{0 n}$ vanishes, and so we already know that the $m=0$ Schrödinger equation is satisfied.

$$
\text { 1. } m=5, n=1
$$

The only contribution

$H_{3}|0\rangle_{1}^{21} \supset \frac{1}{6} \int d x V^{(3)}[\sqrt{\lambda} f(x)] g_{B}^{3}(x) \phi_{0}^{3}|0\rangle_{1}^{21}=0$

vanishes because

$$
\begin{aligned}
V_{B B B} & =\int d x V^{(3)}[\sqrt{\lambda} f(x)] g_{B}^{3}(x)=\frac{1}{\sqrt{Q_{0}}} \int d x\left(\partial_{x} V^{(2)}[\sqrt{\lambda} f(x)]\right) g_{B}^{2}(x), \\
& =-\frac{2}{\sqrt{Q_{0}}} \int d x V^{(2)}[\sqrt{\lambda} f(x)] g_{B}(x) g_{B}^{\prime}(x)=-\frac{2}{\sqrt{Q_{0}}} \int d x g_{B}^{\prime \prime}(x) g_{B}^{\prime}(x)=0
\end{aligned}
$$

is a total derivative.

\footnotetext{
${ }^{6} \mathrm{~A}$ state is BPS if it is annihilated by any nonzero element of the supersymmetry algebra. In this case, the operator is annihilated by half of the fermionic generators.
} 
2. $m=4, n=2$

$$
H_{3}|0\rangle_{1}^{21} \supset \frac{1}{4 \sqrt{Q_{0}}} \int \frac{d^{2} k}{(2 \pi)^{2}} \omega_{k_{1}} \Delta_{k_{1} B} V_{k_{2} B B} \phi_{0}^{4} B_{k_{1}}^{\dagger} B_{k_{2}}^{\dagger}|0\rangle_{0}
$$

exactly cancels

$$
\int \frac{d k^{\prime}}{2 \pi} \omega_{k^{\prime}} B_{k^{\prime}}^{\dagger} B_{k^{\prime}}|0\rangle_{2}^{42}=\frac{1}{4 Q_{0}} \int \frac{d^{2} k}{(2 \pi)^{2}} \omega_{k_{1}}^{2} \omega_{k_{2}} \Delta_{k_{1} B} \Delta_{k_{2} B} \phi_{0}^{4} B_{k_{1}}^{\dagger} B_{k_{2}}^{\dagger}|0\rangle_{0}
$$

as a result of (4.10).

$$
\text { 3. } m=4, n=0
$$

$$
H_{3}|0\rangle_{1}^{21} \supset-\frac{1}{8} \int \frac{d^{1} k}{(2 \pi)^{1}} Y_{k B B} Y_{-k B B} \phi_{0}^{4}|0\rangle_{0}
$$

exactly cancels

$$
H_{4}|0\rangle_{0} \supset \frac{V_{B B B B}}{24} \phi_{0}^{4}|0\rangle_{0}
$$

as

$$
\begin{aligned}
V_{B B B B} & =\int d x V^{(4)}[\sqrt{\lambda} f(x)] \lambda_{B}^{2}(x)=\int d x V^{(4)}[\sqrt{\lambda} f(x)] g_{B}^{3}(x) \frac{f^{\prime}(x)}{\sqrt{Q_{0}}}, \\
& =\frac{1}{\sqrt{Q_{0}}} \int d x \partial_{x}\left(V^{(3)}[\sqrt{\lambda} f(x)]\right) g_{B}^{3}(x)=-\frac{3}{\sqrt{Q_{0}}} \int d x V^{(3)}[\sqrt{\lambda} f(x)] g_{B}^{2}(x) g_{B}^{\prime}(x), \\
& =-\frac{3}{\sqrt{Q_{0}}} \int d x V^{(3)}[\sqrt{\lambda} f(x)] g_{B}^{2}(x) \int d y \delta(x-y) g_{B}^{\prime}(y), \\
& =-\frac{3}{\sqrt{Q_{0}}} \int d x V^{(3)}[\sqrt{\lambda} f(x)] g_{B}^{2}(x) \int d y\left[g_{B}(x) g_{B}(y)+\int \frac{d k}{2 \pi} g_{k}(x) g_{-k}(y)\right] g_{B}^{\prime}(y), \\
& =-\frac{3}{\sqrt{Q_{0}}} \int \frac{d k}{2 \pi} V_{k B B} \Delta_{-k B}=3 \int \frac{d k}{2 \pi} Y_{k B B} Y_{-k B B} .
\end{aligned}
$$

$$
\text { 4. } m=3, n=3
$$

The two terms in

$$
\int \frac{d k^{\prime}}{2 \pi} \omega_{k^{\prime}} B_{k^{\prime}}^{\dagger} B_{k^{\prime}}|0\rangle_{2}^{33}=-\frac{1}{4 \sqrt{Q_{0}}} \int \frac{d^{3} k}{(2 \pi)^{3}}\left[V_{k_{1} k_{2} B} \omega_{k_{3}} \Delta_{k_{3} B}+V_{k_{3} B B}\left(\omega_{k_{1}}-\omega_{k_{2}}\right) \Delta_{k_{1} k_{2}}\right] \phi_{0}^{3} B_{k_{1}}^{\dagger} B_{k_{2}}^{\dagger} B_{k_{3}}^{\dagger}|0\rangle_{0}
$$

are, respectively, canceled by $H_{3}|0\rangle_{1}^{21}$ and $H_{3}|0\rangle_{1}^{12}$. 


\section{5. $m=3, n=1$}

We will need the identity

$$
\begin{aligned}
V_{\mathcal{I B}} & =\int d x V^{(3)}[\sqrt{\lambda} f(x)] g_{B}(x) \mathcal{I}(x)=\frac{1}{\sqrt{Q_{0}}} \int d x\left(\partial_{x} V^{(2)}[\sqrt{\lambda} f(x)]\right) \mathcal{I}(x), \\
& =-\frac{1}{\sqrt{Q_{0}}} \int d x V^{(2)}[\sqrt{\lambda} f(x)] \mathcal{I}^{\prime}(x)=-\frac{1}{\sqrt{Q_{0}}} \int d x V^{(2)}[\sqrt{\lambda} f(x)] \int \frac{d k}{2 \pi} \frac{g_{k}(x) g_{-k}^{\prime}(x)}{\omega_{k}} \\
& =-\frac{1}{\sqrt{Q_{0}}} \int \frac{d k}{2 \pi} \int d x\left(\omega_{k} g_{k}(x)+\frac{g_{k}^{\prime \prime}(x)}{\omega_{k}}\right) g_{-k}^{\prime}(x), \\
& =-\frac{1}{2 \sqrt{Q_{0}}} \int \frac{d k}{2 \pi} \int d x \partial_{x}\left(\omega_{k}\left|g_{k}(x)\right|^{2}+\frac{\left|g_{k}^{\prime}(x)\right|^{2}}{\omega_{k}}\right)=0
\end{aligned}
$$

Note that although this is the integral of a total derivative, the differentiated function does not vanish at infinity. The integral vanishes because the differentiated function is even in $x$. This is true at each $k$ if the potential is symmetric under an inversion that exchanges the two minima responsible for the kink, as it is in the Sine-Gordon model and the $\phi^{4}$ model. More generally, in the large $x$ region which fixes this integral by the fundamental theorem of calculus, the functions $g_{k}(x)$ are plane waves and their norm is constant and independent of the potential. In the case of a reflectionless potential, the norm is equal in both asymptotic regimes and so this integral vanishes for each value of $k$. More generally, the integral vanishes when summed over $k$ and $-k$ as the summed norms squared are equal in the two asymptotic regions.

Using this identity, one evaluates the contribution of $|0\rangle_{1}^{21}$ to be

$$
H_{3}|0\rangle_{1}^{21} \supset \frac{1}{4 \sqrt{Q_{0}}} \int \frac{d k}{2 \pi} \int \frac{d k^{\prime}}{2 \pi} V_{B k-k^{\prime}} \Delta_{k^{\prime} B} \phi_{0}^{3} B_{k}^{\dagger}|0\rangle_{0} .
$$

Similarly

$$
\begin{aligned}
& H_{3}|0\rangle_{1}^{12} \supset \frac{1}{4 \sqrt{Q_{0}}} \int \frac{d k}{2 \pi} \int \frac{d k^{\prime}}{2 \pi}\left(\frac{\omega_{k}}{\omega_{k^{\prime}}}-1\right) V_{B B-k^{\prime}} \Delta_{k k^{\prime}} \phi_{0}^{3} B_{k}^{\dagger}|0\rangle_{0}, \\
& \int \frac{d k^{\prime}}{2 \pi} \omega_{k^{\prime}} B_{k^{\prime}}^{\dagger} B_{k^{\prime}}|0\rangle_{2}^{31}=\frac{1}{12 Q_{0}} \int \frac{d k}{2 \pi} \int \frac{d k^{\prime}}{2 \pi}\left(3 \omega_{k^{\prime}}-\omega_{k}\right) \omega_{k} \Delta_{-k^{\prime} B} \Delta_{k k^{\prime}} \phi_{0}^{3} B_{k}^{\dagger}|0\rangle_{0} .
\end{aligned}
$$

The final contribution is

$$
H_{4}|0\rangle_{0}=\frac{1}{6} \int \frac{d k}{2 \pi} V_{B B B k} \phi_{0}^{3} B_{k}^{\dagger}|0\rangle_{0}
$$

The $V$ s may all be traded for $\Delta$ susing (4.10) and, as may be derived similarly to (A7),

$$
\begin{aligned}
V_{B B B k}= & \frac{1}{\sqrt{Q_{0}}} \int d x \partial_{x}\left(V^{(3)}[\sqrt{\lambda} f(x)]\right) g_{B}(x)^{2} g_{k}(x), \\
= & -\frac{1}{\sqrt{Q_{0}}} \int d x V^{(3)}[\sqrt{\lambda} f(x)]\left[2 g_{B}(x) g_{k}(x) \int d y\left(g_{B}(x) g_{B}(y)+\int \frac{d k^{\prime}}{2 \pi} g_{k^{\prime}}(x) g_{-k^{\prime}}(y)\right) g_{B}^{\prime}(y)\right. \\
& \left.+g_{B}^{2}(x) \int d y\left(g_{B}(x) g_{B}(y)+\int \frac{d k^{\prime}}{2 \pi} g_{k^{\prime}}(x) g_{-k^{\prime}}(y)\right) g_{k}^{\prime}(y)\right], \\
= & -\frac{1}{\sqrt{Q_{0}}} \int \frac{d k^{\prime}}{2 \pi}\left(2 V_{B k k^{\prime}} \Delta_{-k^{\prime} B}+V_{B B k^{\prime}} \Delta_{-k^{\prime} k}\right)=\frac{1}{Q_{0}} \int \frac{d k^{\prime}}{2 \pi}\left(2 \omega_{k}^{2}-3 \omega_{k^{\prime}}^{2}\right) \Delta_{k k^{\prime}} \Delta_{-k^{\prime} B} .
\end{aligned}
$$

Combining these contributions 


$$
\begin{aligned}
\Gamma_{2}^{31}(k) & =\int \frac{d k^{\prime}}{2 \pi} \Delta_{k k^{\prime}} \Delta_{-k^{\prime} B}\left(\frac{\omega_{k^{\prime}}^{2}-\omega_{k}^{2}}{4}-\frac{\omega_{k^{\prime}}^{2}}{4}\left(\frac{\omega_{k}}{\omega_{k^{\prime}}}-1\right)+\frac{3 \omega_{k^{\prime}} \omega_{k}-\omega_{k}^{2}}{12}+\frac{2 \omega_{k}^{2}-3 \omega_{k^{\prime}}^{2}}{6}\right), \\
& =0 .
\end{aligned}
$$

$$
\text { 6. } m=2, n=4
$$

The contributions are

$$
\begin{aligned}
& H_{3}|0\rangle_{1}^{21} \supset \frac{1}{12 \sqrt{Q_{0}}} \int \frac{d^{4} k}{(2 \pi)^{4}} \omega_{k_{1}} \Delta_{k_{1} B} V_{k_{2} k_{3} k_{4}} \phi_{0}^{2} B_{k_{1}}^{\dagger} B_{k_{2}}^{\dagger} B_{k_{3}}^{\dagger} B_{k_{4}}^{\dagger}|0\rangle_{0}, \\
& H_{3}|0\rangle_{1}^{12} \supset \frac{1}{4 \sqrt{Q_{0}}} \int \frac{d^{4} k}{(2 \pi)^{4}}\left(\omega_{k_{1}}-\omega_{k_{2}}\right) \Delta_{k_{1} k_{2}} V_{B k_{3} k_{4}} \phi_{0}^{2} B_{k_{1}}^{\dagger} B_{k_{2}}^{\dagger} B_{k_{3}}^{\dagger} B_{k_{4}}^{\dagger}|0\rangle_{0}, \\
& H_{3}|0\rangle_{1}^{03} \supset-\frac{1}{12} \int \frac{d^{4} k}{(2 \pi)^{4}} V_{B B k_{1}} Y_{k_{2} k_{3} k_{4}} \phi_{0}^{2} B_{k_{1}}^{\dagger} B_{k_{2}}^{\dagger} B_{k_{3}}^{\dagger} B_{k_{4}}^{\dagger}|0\rangle_{0},
\end{aligned}
$$

and

$$
\int \frac{d k^{\prime}}{2 \pi} \omega_{k^{\prime}} B_{k^{\prime}}^{\dagger} B_{k^{\prime}}|0\rangle_{2}^{24}=\int \frac{d^{4} k}{(2 \pi)^{4}}\left(\frac{Y_{B k_{1} k_{2}} V_{B k_{3} k_{4}}}{4}-\Omega_{4} \frac{\omega_{k_{1}} \Delta_{k_{1} B} Y_{k_{2} k_{3} k_{4}}}{12 \sqrt{Q_{0}}}\right) \phi_{0}^{2} B_{k_{1}}^{\dagger} B_{k_{2}}^{\dagger} B_{k_{3}}^{\dagger} B_{k_{4}}^{\dagger}|0\rangle_{0} .
$$

Therefore

$$
\begin{aligned}
\Gamma_{2}^{24}\left(k_{1} \cdots k_{4}\right)= & \frac{\sqrt{Q_{0}} \Delta_{k_{1} B} V_{k_{2} k_{3} k_{4}}}{12}\left[\omega_{k_{1}}+\frac{\omega_{k_{1}}^{2}}{\omega_{k_{2}}+\omega_{k_{3}}+\omega_{k_{4}}}-\frac{\omega_{k_{1}} \Omega_{4}}{\omega_{k_{2}}+\omega_{k_{3}}+\omega_{k_{4}}}\right] \\
& +\frac{\sqrt{Q_{0}} \Delta_{k_{1} k_{2}} V_{B k_{3} k_{4}}}{4}\left[\left(\omega_{k_{1}}-\omega_{k_{2}}\right)+\frac{\omega_{k_{2}}^{2}-\omega_{k_{1}}^{2}}{\omega_{k_{1}}+\omega_{k_{2}}}\right]=0
\end{aligned}
$$

as the terms in each square bracket vanish.

$$
\text { 7. } m=2, n=2
$$

From here on there will be many more contributions to each $\Gamma_{2}^{m n}$, and so we will decompose them into pieces that are not expected to mix as was done in Sec. IV B. First let us consider contributions that depend on $\mathcal{I}(x)$ and so on our renormalization scheme. As we have seen that $V_{\mathcal{I} B}$ vanishes, there are three contributions

$$
\begin{aligned}
& H_{3}|0\rangle_{1}^{21} \supset \frac{1}{4 \sqrt{Q_{0}}} \int \frac{d^{2} k}{(2 \pi)^{2}} V_{\mathcal{I k}_{1}} \omega_{k_{2}} \Delta_{k_{2} B} \phi_{0}^{2} B_{k_{1}}^{\dagger} B_{k_{2}}^{\dagger}|0\rangle_{0}, \\
& H_{3}|0\rangle_{1}^{01} \supset \frac{1}{4 \sqrt{Q_{0}}} \int \frac{d^{2} k}{(2 \pi)^{2}} Y_{\mathcal{I} k_{1}} \omega_{k_{2}}^{2} \Delta_{k_{2} B} \phi_{0}^{2} B_{k_{1}}^{\dagger} B_{k_{2}}^{\dagger}|0\rangle_{0}, \\
& \int \frac{d k^{\prime}}{2 \pi} \omega_{k^{\prime}} B_{k^{\prime}}^{\dagger} B_{k^{\prime}}|0\rangle_{2}^{22} \supset-\frac{1}{4 \sqrt{Q_{0}}} \int \frac{d^{2} k}{(2 \pi)^{2}}\left(\omega_{k_{1}}+\omega_{k_{2}}\right) Y_{\mathcal{I} k_{1}} \omega_{k_{2}} \omega_{k_{2}}^{2} \Delta_{k_{2} B} \phi_{0}^{2} B_{k_{1}}^{\dagger} B_{k_{2}}^{\dagger}|0\rangle_{0},
\end{aligned}
$$

whose sum is easily seen to vanish.

Similarly to (A13) one may derive

$$
V_{B B k_{1} k_{2}}=\frac{1}{Q_{0}}\left[-\left(\omega_{k_{1}}^{2}+\omega_{k_{2}}^{2}\right) \Delta_{k_{1} B} \Delta_{k_{2} B}+\int \frac{d k^{\prime}}{2 \pi}\left[-\sqrt{Q_{0}} V_{k_{1} k_{2} k^{\prime}} \Delta_{-k^{\prime} B}+\left(\omega_{k_{1}}^{2}+\omega_{k_{2}}^{2}-2 \omega_{k^{\prime}}^{2}\right) \Delta_{k_{2} k^{\prime}} \Delta_{-k^{\prime} k_{1}}\right]\right] .
$$

There are four terms that contain $V_{k_{1} k_{2} k_{3}}$ 


$$
\begin{aligned}
H_{4}|0\rangle_{0} & \supset-\frac{1}{4 \sqrt{Q_{0}}} \int \frac{d^{2} k}{(2 \pi)^{2}} \int \frac{d k^{\prime}}{2 \pi} V_{k_{1} k_{2} k^{\prime}} \Delta_{-k^{\prime} B} \phi_{0}^{2} B_{k_{1}}^{\dagger} B_{k_{2}}^{\dagger}|0\rangle_{0}, \\
H_{3}|0\rangle_{1}^{03} & \supset \frac{1}{8 \sqrt{Q_{0}}} \int \frac{d^{2} k}{(2 \pi)^{2}} \int \frac{d k^{\prime}}{2 \pi} Y_{k_{1} k_{2} k^{\prime}} \omega_{k^{\prime}} \Delta_{-k^{\prime} B} \phi_{0}^{2} B_{k_{1}}^{\dagger} B_{k_{2}}^{\dagger}|0\rangle_{0}, \\
H_{3}|0\rangle_{1}^{21} & \supset \frac{1}{8 \sqrt{Q_{0}}} \int \frac{d^{2} k}{(2 \pi)^{2}} \int \frac{d k^{\prime}}{2 \pi} V_{k_{1} k_{2} k^{\prime}} \Delta_{-k^{\prime} B} \phi_{0}^{2} B_{k_{1}}^{\dagger} B_{k_{2}}^{\dagger}|0\rangle_{0}, \\
\int \frac{d k^{\prime}}{2 \pi} \omega_{k^{\prime}} B_{k^{\prime}}^{\dagger} B_{k^{\prime}}|0\rangle_{2}^{22} & \supset \frac{1}{8 \sqrt{Q_{0}}} \int \frac{d^{2} k}{(2 \pi)^{2}} \int \frac{d k^{\prime}}{2 \pi}\left(\omega_{k_{1}}+\omega_{k_{2}}\right) Y_{k_{1} k_{2} k^{\prime}} \Delta_{-k^{\prime} B} \phi_{0}^{2} B_{k_{1}}^{\dagger} B_{k_{2}}^{\dagger}|0\rangle_{0},
\end{aligned}
$$

which again sum to zero, as the second plus the fourth and also the third are equal to minus one half of the first. The four terms with no $k^{\prime}$ integral are

$$
\begin{aligned}
H_{4}|0\rangle_{0} & \supset-\frac{1}{4 Q_{0}} \int \frac{d^{2} k}{(2 \pi)^{2}}\left(\omega_{k_{1}}^{2}+\omega_{k_{2}}^{2}\right) \Delta_{k_{1} B} \Delta_{k_{2} B} \phi_{0}^{2} B_{k_{1}}^{\dagger} B_{k_{2}}^{\dagger}|0\rangle_{0}, \\
H_{3}|0\rangle_{1}^{01} & \supset-\frac{1}{8 Q_{0}} \int \frac{d^{2} k}{(2 \pi)^{2}}\left(\omega_{k_{1}}^{2}+\omega_{k_{2}}^{2}\right) \Delta_{k_{1} B} \Delta_{k_{2} B} \phi_{0}^{2} B_{k_{1}}^{\dagger} B_{k_{2}}^{\dagger}|0\rangle_{0}, \\
\frac{\pi_{0}^{2}}{2}|0\rangle_{2}^{42} & =-\frac{3}{4 Q_{0}} \int \frac{d^{2} k}{(2 \pi)^{2}} \omega_{k_{1}} \omega_{k_{2}} \Delta_{k_{1} B} \Delta_{k_{2} B} \phi_{0}^{2} B_{k_{1}}^{\dagger} B_{k_{2}}^{\dagger}|0\rangle_{0}, \\
\int \frac{d k^{\prime}}{2 \pi} \omega_{k^{\prime}} B_{k^{\prime}}^{\dagger} B_{k^{\prime}}|0\rangle_{2}^{22} & \supset \frac{3}{8 Q_{0}} \int \frac{d^{2} k}{(2 \pi)^{2}} \Omega_{2}^{2} \Delta_{k_{1} B} \Delta_{k_{2} B} \phi_{0}^{2} B_{k_{1}}^{\dagger} B_{k_{2}}^{\dagger}|0\rangle_{0},
\end{aligned}
$$

which sum easily to zero as well. Finally the three terms with $k^{\prime}$ but no $V_{k_{1} k_{2} k_{3}}$ are

$$
\begin{gathered}
H_{4}|0\rangle_{0} \supset \frac{1}{4 Q_{0}} \int \frac{d^{2} k}{(2 \pi)^{2}} \int \frac{d k^{\prime}}{2 \pi}\left(\omega_{k_{1}}^{2}+\omega_{k_{2}}^{2}-2 \omega_{k^{\prime}}^{2}\right) \Delta_{k_{1} k^{\prime}} \Delta_{-k^{\prime} k_{2}} \phi_{0}^{2} B_{k_{1}}^{\dagger} B_{k_{2}}^{\dagger}|0\rangle_{0}, \\
H_{3}|0\rangle_{1}^{12} \supset \frac{1}{2 Q_{0}} \int \frac{d^{2} k}{(2 \pi)^{2}} \int \frac{d k^{\prime}}{2 \pi}\left(\frac{\omega_{k_{2}}^{2}}{\omega_{k^{\prime}}}-\omega_{k^{\prime}}\right)\left(\omega_{k_{1}}-\omega_{k^{\prime}}\right) \Delta_{k_{1} k^{\prime}} \Delta_{-k^{\prime} k_{2}} \phi_{0}^{2} B_{k_{1}}^{\dagger} B_{k_{2}}^{\dagger}|0\rangle_{0}, \\
\int \frac{d k^{\prime}}{2 \pi} \omega_{k^{\prime}} B_{k^{\prime}}^{\dagger} B_{k^{\prime}}|0\rangle_{2}^{22} \supset-\frac{1}{4 Q_{0}} \int \frac{d^{2} k}{(2 \pi)^{2}} \int \frac{d k^{\prime}}{2 \pi} \Omega_{2}\left(\frac{\omega_{k_{1}} \omega_{k_{2}}}{\omega_{k^{\prime}}}-\omega_{k^{\prime}}\right) \Delta_{k_{1} k^{\prime}} \Delta_{-k^{\prime} k_{2}} \phi_{0}^{2} B_{k_{1}}^{\dagger} B_{k_{2}}^{\dagger}|0\rangle_{0} .
\end{gathered}
$$

Notice that in the second term, all factors are symmetric with respect to $k_{1} \leftrightarrow k_{2}$ except for the factors of $\omega$. Therefore these may be symmetrized to

$$
\frac{1}{2}\left(\frac{\omega_{k_{1}} \omega_{k_{2}} \Omega_{2}}{\omega_{k^{\prime}}}-\omega_{k_{1}}^{2}-\omega_{k_{2}}^{2}-\omega_{k^{\prime}} \Omega_{2}\right)+\omega_{k^{\prime}}^{2}
$$

which exactly cancels the corresponding contributions from the first and third terms. We thus conclude that $\Gamma_{2}^{22}=0$.

\section{8. $m=2, n=0$}

We will see that this is the most interesting case so far, because it is the first that strongly depends on the form of $\mathcal{I}(x)$. To see this, let us try to proceed as above. The terms that depend on $\mathcal{I}(x)$ are

$$
\begin{aligned}
& H_{4}|0\rangle_{0} \supset \frac{1}{4} V_{\mathcal{I} B B} \phi_{0}^{2}|0\rangle_{0}, \\
& H_{3}|0\rangle_{1}^{21} \supset \frac{1}{8 \sqrt{Q_{0}}} \int \frac{d k^{\prime}}{2 \pi} V_{\mathcal{I} k^{\prime}} \Delta_{-k^{\prime} k_{2}} \phi_{0}^{2}|0\rangle_{0}, \\
& H_{3}|0\rangle_{1}^{01} \supset \frac{1}{8 \sqrt{Q_{0}}} \int \frac{d k^{\prime}}{2 \pi} V_{\mathcal{I} k^{\prime}} \Delta_{-k^{\prime} k_{2}} \phi_{0}^{2}|0\rangle_{0} .
\end{aligned}
$$


As above, we may eliminate $V^{(4)}$ using integration by parts and then inserting the completeness relation (2.16)

$$
\begin{aligned}
V_{\mathcal{I} B B} & =\int d x V^{(4)}[\sqrt{\lambda} f(x)] \mathcal{I}(x) g_{B}^{2}(x)=\frac{1}{\sqrt{Q_{0}}} \int d x\left(\partial_{x} V^{(3)}[\sqrt{\lambda} f(x)]\right) \mathcal{I}(x) g_{B}(x), \\
& =-\frac{1}{\sqrt{Q_{0}}} \int d x V^{(3)}[\sqrt{\lambda} f(x)]\left(\mathcal{I}^{\prime}(x) g_{B}(x)+\mathcal{I}(x) g_{B}^{\prime}(x)\right), \\
& =-\frac{1}{\sqrt{Q_{0}}}\left(V_{\mathcal{I}^{\prime} B}+\int \frac{d k^{\prime}}{2 \pi} V_{\mathcal{I} k^{\prime} \Delta_{-k^{\prime} B}}\right) .
\end{aligned}
$$

The second term cancels the contributions from $H_{3}|0\rangle_{1}^{21}$ and $H_{3}|0\rangle_{1}^{01}$, leaving

$$
\Gamma_{2, \mathcal{I}}^{20}=-\frac{\sqrt{Q_{0}}}{4} V_{\mathcal{I}^{\prime} B}
$$

Let us rewrite $V_{\mathcal{I}^{\prime} B}$ in terms of quantities that we expect to find in other contributions.

Writing the identity (2.24) as

$$
\partial_{x} \mathcal{I}(x)=\int \frac{d k}{2 \pi} \frac{1}{2 \omega_{k}}\left(g_{k}(x) g_{-k}^{\prime}(x)+g_{k}^{\prime}(x) g_{-k}(x)\right),
$$

one finds

$$
\begin{aligned}
V_{\mathcal{I}^{\prime} B} & =\int \frac{d k}{2 \pi} \frac{1}{2 \omega_{k}} \int d x V^{(3)}[\sqrt{\lambda} f(x)] g_{B}(x)\left(g_{k}(x) g_{-k}^{\prime}(x)+g_{k}^{\prime}(x) g_{-k}(x)\right), \\
& =\int \frac{d k}{2 \pi} \frac{1}{\omega_{k}}\left(V_{B B k} \Delta_{B k}+\int \frac{d k^{\prime}}{2 \pi} V_{B k k^{\prime}} \Delta_{-k^{\prime} k}\right) \\
& =-\frac{1}{\sqrt{Q_{0}}} \int \frac{d^{2} k^{\prime}}{(2 \pi)^{2}} \frac{\omega_{k_{1}^{\prime}}^{2}-\omega_{k_{2}^{\prime}}^{2}}{\omega_{k_{1}^{\prime}}} \Delta_{k_{1}^{\prime} k_{2}^{\prime}} \Delta_{-k_{1}^{\prime}-k_{2}^{\prime}}-\frac{1}{\sqrt{Q_{0}}} \int \frac{d k^{\prime}}{2 \pi} \omega_{k^{\prime}} \Delta_{B k^{\prime}} \Delta_{-k^{\prime} B}
\end{aligned}
$$

Inserting this into (A26) and symmetrizing dummy indices one finds

$$
\Gamma_{2, \mathcal{I}}^{20}=\frac{1}{8} \int \frac{d^{2} k^{\prime}}{(2 \pi)^{2}}\left(-\omega_{k_{1}^{\prime}}-\omega_{k_{2}^{\prime}}+\frac{\omega_{k_{1}^{\prime}}^{2}}{\omega_{k_{2}^{\prime}}}+\frac{\omega_{k_{2}^{\prime}}^{2}}{\omega_{k_{1}^{\prime}}^{\prime}}\right) \Delta_{k_{1}^{\prime} k_{2}^{\prime}} \Delta_{-k_{1}^{\prime}-k_{2}^{\prime}}+\frac{1}{4} \int \frac{d k^{\prime}}{2 \pi} \omega_{k^{\prime}} \Delta_{B k^{\prime}} \Delta_{-k^{\prime} B} .
$$

Schrödinger's equation will only be satisfied if these terms are canceled by contributions with no $\mathcal{I}(x)$.

There is only one contribution with no $\mathcal{I}(x)$ that has two contracted momenta $k^{\prime}$

$$
H_{3}|0\rangle_{1}^{12} \supset \frac{1}{8 Q_{0}} \int \frac{d^{2} k^{\prime}}{(2 \pi)^{2}}\left(\omega_{k_{1}^{\prime}}+\omega_{k_{2}^{\prime}}-\frac{\omega_{k_{1}^{\prime}}^{2}}{\omega_{k_{2}^{\prime}}}-\frac{\omega_{k_{2}^{\prime}}^{2}}{\omega_{k_{1}^{\prime}}}\right) \Delta_{k_{1}^{\prime} k_{2}^{\prime}} \Delta_{-k_{1}^{\prime}-k_{2}^{\prime}} \phi_{0}^{2}|0\rangle_{0},
$$

which indeed cancels the first term in (A29). There are two contributions with no $\mathcal{I}(x)$ and a single $k^{\prime}$

$$
\begin{aligned}
& H_{3}|0\rangle_{1}^{01} \supset \frac{1}{8 Q_{0}} \int \frac{d k^{\prime}}{2 \pi} \omega_{k^{\prime}} \Delta_{B k^{\prime}} \Delta_{-k^{\prime} B} \phi_{0}^{2}|0\rangle_{0}, \\
& \frac{\pi_{0}^{2}}{2}|0\rangle_{2}^{40}=-\frac{3}{8 Q_{0}} \int \frac{d k^{\prime}}{2 \pi} \omega_{k^{\prime}} \Delta_{B k^{\prime}} \Delta_{-k^{\prime} B} \phi_{0}^{2}|0\rangle_{0},
\end{aligned}
$$

which are equal to $1 / 2$ and $-3 / 2$ of the second term in (A29), and so altogether they cancel, leaving $\Gamma_{2}^{20}=0$. 


\section{9. $m=1, n=5$}

There are only three contributions to this term

$$
\begin{aligned}
H_{3}|0\rangle_{1}^{03} \supset \frac{1}{12 \sqrt{Q_{0}}} \int \frac{d^{5} k}{(2 \pi)^{5}}\left(\omega_{k_{4}}^{2}-\omega_{k_{5}}^{2}\right) Y_{k_{1} k_{2} k_{3}} \Delta_{k_{4} k_{5}} \phi_{0} B_{k_{1}}^{\dagger} \cdots B_{k_{5}}^{\dagger}|0\rangle_{0}, \\
H_{3}|0\rangle_{1}^{12} \supset \frac{1}{12 \sqrt{Q_{0}}} \int \frac{d^{5} k}{(2 \pi)^{5}}\left(\omega_{k_{4}}-\omega_{k_{5}}\right) V_{k_{1} k_{2} k_{3}} \Delta_{k_{4} k_{5}} \phi_{0} B_{k_{1}}^{\dagger} \cdots B_{k_{5}}^{\dagger}|0\rangle_{0}, \\
\int \frac{d k^{\prime}}{2 \pi} \omega_{k^{\prime}} B_{k^{\prime}}^{\dagger} B_{k^{\prime}}|0\rangle_{2}^{15}=-\frac{1}{12 \sqrt{Q_{0}}} \int \frac{d^{5} k}{(2 \pi)^{5}}\left(\omega_{k_{4}}-\omega_{k_{5}}+\frac{\omega_{k_{4}}^{2}-\omega_{k_{5}}^{2}}{\Omega_{3}}\right) V_{k_{1} k_{2} k_{3}} \Delta_{k_{4} k_{5}} \phi_{0} B_{k_{1}}^{\dagger} \cdots B_{k_{5}}^{\dagger}|0\rangle_{0},
\end{aligned}
$$

whose sum is readily seen to vanish.

\section{0. $m=1, n=3$}

Again let us divide the 11 contributions to $\Gamma_{2}^{13}$ into three subsets that are expected to cancel separately. First, terms involving $\mathcal{I}(x)$ are

$$
\begin{aligned}
& H_{3}|0\rangle_{1}^{12} \supset \frac{1}{4 \sqrt{Q_{0}}} \int \frac{d^{3} k}{(2 \pi)^{3}}\left(\omega_{k_{2}}-\omega_{k_{3}}\right) V_{\mathcal{I} k_{1}} \Delta_{k_{2} k_{3}} \phi_{0} B_{k_{1}}^{\dagger} B_{k_{2}}^{\dagger} B_{k_{3}}^{\dagger}|0\rangle_{0}, \\
& H_{3}|0\rangle_{1}^{01} \supset \frac{1}{4 \sqrt{Q_{0}}} \int \frac{d^{3} k}{(2 \pi)^{3}}\left(\omega_{k_{2}}^{2}-\omega_{k_{3}}^{2}\right) Y_{\mathcal{I} k_{1}} \Delta_{k_{2} k_{3}} \phi_{0} B_{k_{1}}^{\dagger} B_{k_{2}}^{\dagger} B_{k_{3}}^{\dagger}|0\rangle_{0}, \\
& \int \frac{d k^{\prime}}{2 \pi} \omega_{k^{\prime}} B_{k^{\prime}}^{\dagger} B_{k^{\prime}}|0\rangle_{2}^{13} \supset-\frac{1}{4 \sqrt{Q_{0}}} \int \frac{d^{3} k}{(2 \pi)^{3}}\left(\omega_{k_{2}}-\omega_{k_{3}}\right) \Omega_{3} Y_{\mathcal{I} k_{1}} \Delta_{k_{2} k_{3}} \phi_{0} B_{k_{1}}^{\dagger} B_{k_{2}}^{\dagger} B_{k_{3}}^{\dagger}|0\rangle_{0},
\end{aligned}
$$

which sum to zero.

We will now need

$$
\begin{aligned}
V_{B k_{1} k_{2} k_{3}}= & \frac{1}{Q_{0}}\left[\left(\omega_{k_{2}}^{2}-\omega_{k_{3}}^{2}\right) \Delta_{B k_{1}} \Delta_{k_{2} k_{3}}+\left(\omega_{k_{1}}^{2}-\omega_{k_{3}}^{2}\right) \Delta_{B k_{2}} \Delta_{k_{1} k_{3}}+\left(\omega_{k_{1}}^{2}-\omega_{k_{2}}^{2}\right) \Delta_{B k_{3}} \Delta_{k_{1} k_{2}}\right] \\
& -\frac{1}{\sqrt{Q_{0}}} \int \frac{d k^{\prime}}{2 \pi}\left[V_{k_{2} k_{3} k^{\prime}} \Delta_{-k^{\prime} k_{1}}+V_{k_{1} k_{3} k^{\prime}} \Delta_{-k^{\prime} k_{2}}+V_{k_{1} k_{2} k^{\prime}} \Delta_{-k^{\prime} k_{3}}\right] .
\end{aligned}
$$

The terms which have a contracted index $k^{\prime}$ are

$$
\begin{aligned}
& H_{4}|0\rangle_{0} \supset-\frac{1}{2 \sqrt{Q_{0}}} \int \frac{d^{3} k}{(2 \pi)^{3}} \int \frac{d k^{\prime}}{2 \pi} V_{k_{1} k_{2} k^{\prime}} \Delta_{-k^{\prime} k_{3}} \phi_{0} B_{k_{1}}^{\dagger} B_{k_{2}}^{\dagger} B_{k_{3}}^{\dagger}|0\rangle_{0}, \\
& H_{3}|0\rangle_{1}^{03} \supset \frac{1}{4 \sqrt{Q_{0}}} \int \frac{d^{3} k}{(2 \pi)^{3}} \int \frac{d k^{\prime}}{2 \pi} \frac{\omega_{k^{\prime}}^{2}-\omega_{k_{3}}^{2}}{\omega_{k^{\prime}}} Y_{k_{1} k_{2} k^{\prime}} \Delta_{-k^{\prime} k_{3}} \phi_{0} B_{k_{1}}^{\dagger} B_{k_{2}}^{\dagger} B_{k_{3}}^{\dagger}|0\rangle_{0}, \\
& H_{3}|0\rangle_{1}^{12} \supset \frac{1}{4 \sqrt{Q_{0}}} \int \frac{d^{3} k}{(2 \pi)^{3}} \int \frac{d k^{\prime}}{2 \pi} \frac{\omega_{k^{\prime}}-\omega_{k_{3}}}{\omega_{k^{\prime}}} V_{k_{1} k_{2} k^{\prime}} \Delta_{-k^{\prime} k_{3}} \phi_{0} B_{k_{1}}^{\dagger} B_{k_{2}}^{\dagger} B_{k_{3}}^{\dagger}|0\rangle_{0}, \\
& \int \frac{d k^{\prime}}{2 \pi} \omega_{k^{\prime}} B_{k^{\prime}}^{\dagger} B_{k^{\prime}}|0\rangle_{2}^{13} \supset \frac{1}{4 \sqrt{Q_{0}}} \int \frac{d^{3} k}{(2 \pi)^{3}} \int \frac{d k^{\prime}}{2 \pi} \Omega_{3} \frac{\omega_{k^{\prime}}+\omega_{k_{3}}}{\omega_{k^{\prime}}} Y_{k_{1} k_{2} k^{\prime}} \Delta_{-k^{\prime} k_{3}} \phi_{0} B_{k_{1}}^{\dagger} B_{k_{2}}^{\dagger} B_{k_{3}}^{\dagger}|0\rangle_{0},
\end{aligned}
$$

whose sum also vanishes. Finally the terms with neither $\mathcal{I}(x)$ nor $k^{\prime}$ are 


$$
\begin{aligned}
& H_{4}|0\rangle_{0} \supset \frac{1}{2 Q_{0}} \int \frac{d^{3} k}{(2 \pi)^{3}}\left(\omega_{k_{2}}^{2}-\omega_{k_{3}}^{2}\right) \Delta_{B k_{1}} \Delta_{k_{2} k_{3}} \phi_{0} B_{k_{1}}^{\dagger} B_{k_{2}}^{\dagger} B_{k_{3}}^{\dagger}|0\rangle_{0}, \\
& H_{3}|0\rangle_{1}^{01} \supset \frac{1}{4 Q_{0}} \int \frac{d^{3} k}{(2 \pi)^{3}}\left(\omega_{k_{2}}^{2}-\omega_{k_{3}}^{2}\right) \Delta_{B k_{1}} \Delta_{k_{2} k_{3}} \phi_{0} B_{k_{1}}^{\dagger} B_{k_{2}}^{\dagger} B_{k_{3}}^{\dagger}|0\rangle_{0}, \\
& \frac{\pi_{0}^{2}}{2}|0\rangle_{2}^{33} \supset \frac{3}{4 Q_{0}} \int \frac{d^{3} k}{(2 \pi)^{3}} \omega_{k_{1}}\left(\omega_{k_{2}}-\omega_{k_{3}}\right) \Delta_{B k_{1}} \Delta_{k_{2} k_{3}} \phi_{0} B_{k_{1}}^{\dagger} B_{k_{2}}^{\dagger} B_{k_{3}}^{\dagger}|0\rangle_{0}, \\
& \int \frac{d k^{\prime}}{2 \pi} \omega_{k^{\prime}} B_{k^{\prime}}^{\dagger} B_{k^{\prime}}|0\rangle_{2}^{13} \supset-\frac{3}{4 Q_{0}} \int \frac{d^{3} k}{(2 \pi)^{3}}\left[\omega_{k_{1}}\left(\omega_{k_{2}}-\omega_{k_{2}}\right)+\omega_{k_{2}}^{2}-\omega_{k_{3}}^{2}\right] \Delta_{B k_{1}} \Delta_{k_{2} k_{3}} \phi_{0} B_{k_{1}}^{\dagger} B_{k_{2}}^{\dagger} B_{k_{3}}^{\dagger}|0\rangle_{0},
\end{aligned}
$$

which again trivially cancel, leaving $\Gamma_{2}^{13}=0$.

\section{1. $m=1, n=1$}

Finally we turn our attention to $\Gamma_{2}^{11}$. Like $\Gamma_{2}^{20}$ we will see that it only vanishes if $\mathcal{I}(x)$ satisfies (2.24). The terms involving $\mathcal{I}(x)$ are

$$
\begin{aligned}
& H_{4}|0\rangle_{0} \supset \frac{1}{2} \int \frac{d k}{2 \pi} V_{\mathcal{I B k}} \phi_{0} B_{k}^{\dagger}|0\rangle_{0}, \\
& H_{3}|0\rangle_{1}^{12} \supset \frac{1}{4 \sqrt{Q_{0}}} \int \frac{d k}{2 \pi} \int \frac{d k^{\prime}}{2 \pi}\left(\omega_{k^{\prime}}-\omega_{k}\right) Y_{\mathcal{I} k^{\prime}} \Delta_{-k^{\prime} k} \phi_{0} B_{k}^{\dagger}|0\rangle_{0}, \\
& H_{3}|0\rangle_{1}^{01} \supset \frac{1}{4 \sqrt{Q_{0}}} \int \frac{d k}{2 \pi} \int \frac{d k^{\prime}}{2 \pi}\left(\frac{\omega_{k^{\prime}}^{2}-\omega_{k}^{2}}{\omega_{k^{\prime}}}\right) Y_{\mathcal{I} k^{\prime}} \Delta_{-k^{\prime} k} \phi_{0} B_{k}^{\dagger}|0\rangle_{0}, \\
& \int \frac{d k^{\prime}}{2 \pi} \omega_{k^{\prime}} B_{k^{\prime}}^{\dagger} B_{k^{\prime}}|0\rangle_{2}^{11} \supset \frac{1}{4 \sqrt{Q_{0}}} \int \frac{d k}{2 \pi} \int \frac{d k^{\prime}}{2 \pi} \omega_{k}\left(\frac{\omega_{k^{\prime}}+\omega_{k}}{\omega_{k^{\prime}}}\right) Y_{\mathcal{I} k^{\prime}} \Delta_{-k^{\prime} k} \phi_{0} B_{k}^{\dagger}|0\rangle_{0} .
\end{aligned}
$$

Again, as in the case of $\Gamma_{2}^{20}$ integration by parts allows us to remove the fourth derivative

$$
V_{\mathcal{I} B k}=-\frac{1}{\sqrt{Q_{0}}} \int d x V^{(3)}[\sqrt{\lambda} f(x)]\left(\mathcal{I}^{\prime}(x) g_{k}(x)+\mathcal{I}(x) g_{k}^{\prime}(x)\right)
$$

and the two terms can be simplified using completeness (2.16) and the formula (2.24) for $\mathcal{I}^{\prime}(x)$

$$
\begin{aligned}
-\frac{1}{\sqrt{Q_{0}}} \int d x V^{(3)}[\sqrt{\lambda} f(x)] \mathcal{I}^{\prime}(x) g_{k}(x)= & \frac{1}{Q_{0}} \int \frac{d k^{\prime}}{2 \pi}\left(\frac{\omega_{k^{\prime}}^{2}-\omega_{k}^{2}}{\omega_{k^{\prime}}}\right) \Delta_{k k^{\prime}} \Delta_{-k^{\prime} B} \\
& -\frac{1}{2 \sqrt{Q_{0}}} \int \frac{d^{2} k^{\prime}}{(2 \pi)^{2}}\left(\frac{\omega_{k_{1}^{\prime}}-\omega_{k_{2}^{\prime}}}{\omega_{k_{1}^{\prime}} \omega_{k_{2}^{\prime}}}\right) V_{k k_{1}^{\prime} k_{2}^{\prime}} \Delta_{-k_{1}^{\prime}-k_{2}^{\prime}}, \\
& -\frac{1}{\sqrt{Q_{0}}} \int d x V^{(3)}[\sqrt{\lambda} f(x)] \mathcal{I}(x) g_{k}^{\prime}(x)=-\frac{1}{\sqrt{Q_{0}}} V_{\mathcal{I} k^{\prime}} \Delta_{-k^{\prime} k} .
\end{aligned}
$$

The second equation in (A39) substituted into the first term in (A37) cancels the second, third, and fourth terms. This leaves only the first equation in (A39), which when substituted into (A37) yields

$$
\Gamma_{2, \mathcal{I}}^{11}=\frac{1}{2} \int \frac{d k^{\prime}}{2 \pi}\left(\frac{\omega_{k^{\prime}}^{2}-\omega_{k}^{2}}{\omega_{k^{\prime}}}\right) \Delta_{k k^{\prime}} \Delta_{-k^{\prime} B}-\frac{\sqrt{Q_{0}}}{4} \int \frac{d^{2} k^{\prime}}{(2 \pi)^{2}}\left(\frac{\omega_{k_{1}^{\prime}}-\omega_{k_{2}^{\prime}}}{\omega_{k_{1}^{\prime}} \omega_{k_{2}^{\prime}}}\right) V_{k k_{1}^{\prime} k_{2}^{\prime}} \Delta_{-k_{1}^{\prime}-k_{2}^{\prime}}
$$


Summing the three contributions with integrals over $k_{1}^{\prime}$ and $k_{2}^{\prime}$

$$
\begin{aligned}
& H_{3}|0\rangle_{1}^{03} \supset \frac{1}{8 \sqrt{Q_{0}}} \int \frac{d k}{2 \pi} \int \frac{d^{2} k^{\prime}}{(2 \pi)^{2}}\left(\frac{\omega_{k_{1}^{\prime}}^{2}-\omega_{k_{2}^{\prime}}^{2}}{\omega_{k_{1}^{\prime}} \omega_{k_{2}^{\prime}}}\right) Y_{k k_{1}^{\prime} k_{2}^{\prime}} \Delta_{-k_{1}^{\prime}-k_{2}^{\prime}} \phi_{0} B_{k}^{\dagger}|0\rangle_{0}, \\
& H_{3}|0\rangle_{1}^{12} \supset \frac{1}{8 \sqrt{Q_{0}}} \int \frac{d k}{2 \pi} \int \frac{d^{2} k^{\prime}}{(2 \pi)^{2}}\left(\frac{\omega_{k_{1}^{\prime}}-\omega_{k_{2}^{\prime}}}{\omega_{k_{1}^{\prime}} \omega_{k_{2}^{\prime}}}\right) V_{k k_{1}^{\prime} k_{2}^{\prime}} \Delta_{-k_{1}^{\prime}-k_{2}^{\prime}} \phi_{0} B_{k}^{\dagger}|0\rangle_{0}, \\
& \int \frac{d k^{\prime}}{2 \pi} \omega_{k^{\prime}} B_{k^{\prime}}^{\dagger} B_{k^{\prime}}|0\rangle_{2}^{11} \supset \frac{1}{8 \sqrt{Q_{0}}} \int \frac{d k}{2 \pi} \int \frac{d^{2} k^{\prime}}{(2 \pi)^{2}} \omega_{k_{1}}\left(\frac{\omega_{k_{1}^{\prime}}-\omega_{k_{2}^{\prime}}}{\omega_{k_{1}^{\prime}} \omega_{k_{2}^{\prime}}}\right) Y_{k k_{1}^{\prime} k_{2}^{\prime}} \Delta_{-k_{1}^{\prime}-k_{2}^{\prime}} \phi_{0} B_{k}^{\dagger}|0\rangle_{0},
\end{aligned}
$$

one obtains

$$
\Gamma_{2,2 k^{\prime}}^{11}=\frac{\sqrt{Q_{0}}}{4} \int \frac{d^{2} k^{\prime}}{(2 \pi)^{2}}\left(\frac{\omega_{k_{1}^{\prime}}-\omega_{k_{2}^{\prime}}}{\omega_{k_{1}^{\prime}} \omega_{k_{2}^{\prime}}}\right) V_{k k_{1}^{\prime} k_{2}^{\prime}} \Delta_{-k_{1}^{\prime}-k_{2}^{\prime}},
$$

which cancels the second term in $\Gamma_{2, \mathcal{I}}^{11}$.

Finally the terms with no $\mathcal{I}(x)$ and one $k^{\prime}$ are

$$
\begin{gathered}
H_{3}|0\rangle_{1}^{01} \supset \frac{1}{4 Q_{0}} \int \frac{d k}{2 \pi} \int \frac{d k^{\prime}}{2 \pi}\left(\omega_{k^{\prime}}-\frac{\omega_{k}^{2}}{\omega_{k^{\prime}}}\right) \Delta_{k k^{\prime}} \Delta_{-k^{\prime} B} \phi_{0} B_{k}^{\dagger}|0\rangle_{0}, \\
\frac{\pi_{0}^{2}}{2}|0\rangle_{2}^{31} \supset \frac{1}{4 Q_{0}} \int \frac{d k}{2 \pi} \int \frac{d k^{\prime}}{2 \pi}\left(\omega_{k}-3 \omega_{k^{\prime}}\right) \Delta_{k k^{\prime}} \Delta_{-k^{\prime} B} \phi_{0} B_{k}^{\dagger}|0\rangle_{0}, \\
\int \frac{d k^{\prime}}{2 \pi} \omega_{k^{\prime}} B_{k^{\prime}}^{\dagger} B_{k^{\prime}}|0\rangle_{2}^{11} \supset \frac{1}{4 Q_{0}} \int \frac{d k}{2 \pi} \int \frac{d k^{\prime}}{2 \pi}\left(-\omega_{k}+3 \frac{\omega_{k}^{2}}{\omega_{k^{\prime}}}\right) \Delta_{k k^{\prime}} \Delta_{-k^{\prime} B} \phi_{0} B_{k}^{\dagger}|0\rangle_{0},
\end{gathered}
$$

which sum to

$$
\Gamma_{2,1 k^{\prime}}^{11}=\frac{1}{2} \int \frac{d k^{\prime}}{2 \pi}\left(\frac{\omega_{k}^{2}-\omega_{k^{\prime}}^{2}}{\omega_{k^{\prime}}}\right) \Delta_{k k^{\prime}} \Delta_{-k^{\prime} B}
$$

canceling the first term in $\Gamma_{2, \mathcal{I}}^{11}$. Summarizing, we have verified that

$$
\Gamma_{2}^{11}=\Gamma_{2, \mathcal{I}}^{11}+\Gamma_{2,1 k^{\prime}}^{11}+\Gamma_{2,2 k^{\prime}}^{11}=0,
$$

and so the state $|0\rangle_{2}$ that we have found indeed solves Schrödinger's equation at two loops.

\section{APPENDIX B: THE MESON MASS IN THE SINE-GORDON MODEL}

In this appendix we briefly review the Schrödinger picture derivation of the two-loop meson mass in the normal-ordered Sine-Gordon model. First one expands the scalar field in terms of Heisenberg operators

$$
\phi(x)=\int \frac{d p}{2 \pi}\left(A_{p}^{\dagger}+\frac{A_{-p}}{2 \omega_{p}}\right) e^{-i p x} .
$$

The Sine-Gordon potential

$$
V(x)=\frac{M^{2}}{\lambda}\left(1-: \cos (\sqrt{\lambda} \phi(x)):_{a}\right)
$$

at fourth order is the interaction

$$
H_{4}=-\frac{M^{2} \lambda}{24} \int d x: \phi^{4}(x):{ }_{a}
$$


while the free Hamiltonian is

$$
H_{2}=\int \frac{d p}{2 \pi} \omega_{p} A_{p}^{\dagger} A_{p} .
$$

Let the meson state $|p\rangle$ be an eigenstate of the full Hamiltonian. Expand it in powers of $\sqrt{\lambda}$

$$
|p\rangle=\sum_{n=0}^{\infty}|p\rangle_{n}
$$

where

$$
|p\rangle_{0}=A_{p}^{\dagger}|\Omega\rangle .
$$

The tree-level Schrödinger equation

$$
H_{2}|p\rangle_{0}=E_{0}|p\rangle_{0}
$$

is solved by $E_{0}=\omega_{p}$. At one loop

$$
0=\left(H_{4}-E_{1}\right)|p\rangle_{0}+\left(H_{2}-E_{0}\right)|p\rangle_{1}
$$

together with the convention ${ }^{7}$

$$
{ }_{0}\langle p \mid p\rangle_{i}=\delta_{0 i}
$$

are solved by $E_{1}=0$ and

$$
\begin{aligned}
|p\rangle_{1}= & \frac{M^{2} \lambda}{24} \int d x \int \frac{d^{4} q}{(2 \pi)^{4}} \frac{e^{-i x \sum_{j}^{4} q_{j}}}{\sum_{j}^{4} \omega_{q_{j}}} A_{q_{1}}^{\dagger} \cdots A_{q_{4}}^{\dagger} A_{p}^{\dagger}|\Omega\rangle \\
& +\frac{M^{2} \lambda}{12} \int d x \int \frac{d^{3} q}{(2 \pi)^{3}} \frac{e^{-i x\left(-p+\sum_{j}^{3} q_{j}\right)}}{\omega_{p}\left(-\omega_{p}+\sum_{j}^{3} \omega_{q_{j}}\right)} \\
& \times A_{q_{1}}^{\dagger} \cdots A_{q_{3}}^{\dagger}|\Omega\rangle .
\end{aligned}
$$

At two loops, the Schrödinger equation is

$$
0=\left(H_{6}-E_{2}\right)|p\rangle_{0}+\left(H_{4}-E_{1}\right)|p\rangle_{1}+\left(H_{2}-E_{0}\right)|p\rangle_{2}
$$

Let us left multiply ${ }_{0}\langle p|$ and use the orthogonality condition (B9). As $H_{6}$ is normal ordered, its matrix element vanishes and one finds

$$
E_{2}={ }_{0}\left\langle p\left|H_{4}\right| p\right\rangle_{1}=A+B,
$$

where

$$
\begin{aligned}
A & =-\frac{M^{4} \lambda^{2}}{48 \omega_{p}} \int \frac{d^{2} q}{(2 \pi)^{2}} \frac{\omega_{q_{1}}+\omega_{q_{2}}+\omega_{p-q_{1}-q_{2}}}{\omega_{q_{1}} \omega_{q_{2}} \omega_{p-q_{1}-q_{2}}\left[\left(\omega_{q_{1}}+\omega_{q_{2}}+\omega_{p-q_{1}-q_{2}}\right)^{2}-\omega_{p}^{2}\right]}, \\
B & =-\frac{M^{4} \lambda^{2}}{384} \int \frac{d^{3} q}{(2 \pi)^{3}} \frac{1}{\omega_{q_{1}} \omega_{q_{2}} \omega_{q_{3}} \omega_{q_{1}+q_{2}+q_{3}}\left(\omega_{q_{1}+q_{2}+q_{3}}+\sum_{i}^{3} \omega_{q_{i}}\right)} .
\end{aligned}
$$

The infrared divergent term $B$ is equal to the two-loop energy of the vacuum state $|\Omega\rangle$ and so it does not contribute to the meson mass. Therefore the two-loop meson mass correction $M_{2}$ is equal to $A$ evaluated at $p=0$

$$
M_{2}=-\frac{M^{3} \lambda^{2}}{48} \int \frac{d^{2} q}{(2 \pi)^{2}} \frac{\omega_{q_{1}}+\omega_{q_{2}}+\omega_{q_{1}+q_{2}}}{\omega_{q_{1}} \omega_{q_{2}} \omega_{q_{1}+q_{2}}\left[\left(\omega_{q_{1}}+\omega_{q_{2}}+\omega_{q_{1}+q_{2}}\right)^{2}-M^{2}\right]}=-\frac{M \lambda^{2}}{768}
$$

in agreement with the pole mass in Ref. [18].

\footnotetext{
${ }^{7}$ For simplicity we have fixed $p$ and applied a $p$-dependent normalization condition on $|\Omega\rangle$.
}

[1] R. F. Dashen, B. Hasslacher, and A. Neveu, Nonperturbative methods and extended hadron models in field theory 1. Semiclassical functional methods, Phys. Rev. D 10, 4114 (1974).

[2] A. Rebhan and P. van Nieuwenhuizen, No saturation of the quantum Bogomolnyi bound by two-dimensional supersymmetric solitons, Nucl. Phys. B508, 449 (1997).

[3] A. Alonso Izquierdo, W. Garcia Fuertes, M. A. Gonzalez Leon, M. de la Torre Mayado, J. Mateos Guilarte, and J. M. Munoz Castaneda, Lectures on the mass of topological solitons, arXiv:hep-th/0611180. 
[4] N. Graham and R. L. Jaffe, Unambiguous one loop quantum energies of $(1+1)$-dimensional bosonic field configurations, Phys. Lett. B 435, 145 (1998).

[5] H. Nastase, M. A. Stephanov, P. van Nieuwenhuizen, and A. Rebhan, Topological boundary conditions, the BPS bound, and elimination of ambiguities in the quantum mass of solitons, Nucl. Phys. B542, 471 (1999).

[6] A. Litvintsev and P. van Nieuwenhuizen, Once more on the BPS bound for the SUSY kink, arXiv:hep-th/0010051.

[7] A. S. Goldhaber, A. Litvintsev, and P. van Nieuwenhuizen, Local Casimir energy for solitons, Phys. Rev. D 67, 105021 (2003).

[8] K. Hepp, The classical limit for quantum mechanical correlation functions, Commun. Math. Phys. 35, 265 (1974).

[9] D. M. A. Stuart, Hamiltonian quantization of solitons in the $\phi_{1+1}^{4}$ quantum field theory. I. The semiclassical mass shift, arXiv:1904.02588.

[10] J. Evslin, Manifestly finite derivation of the quantum Kink mass, J. High Energy Phys. 11 (2019) 161.

[11] H. Guo and J. Evslin, Finite derivation of the one-loop SineGordon soliton mass, J. High Energy Phys. 02 (2020) 140.

[12] J. Evslin, Constructing quantum soliton states despite zero modes, arXiv:2006.02354.

[13] K. E. Cahill, A. Comtet, and R. J. Glauber, Mass formulas for static solitons, Phys. Lett. 64B, 283 (1976).

[14] J. Evslin, Well-defined quantum soliton masses without supersymmetry, Phys. Rev. D 101, 065005 (2020).

[15] J. Goldstone and R. Jackiw, Quantization of nonlinear waves, Phys. Rev. D 11, 1486 (1975).

[16] J. Evslin, Normal ordering normal modes, Eur. Phys. J. C 81, 92 (2021).

[17] J. Evslin and H. Guo, Excited kinks as quantum states, arXiv:2104.03612.

[18] H. J. de Vega, Two-loop quantum corrections to the soliton mass in two-dimensional scalar field theories, Nucl. Phys. B115, 411 (1976).

[19] J. Verwaest, Higher order correction to the Sine-Gordon soliton mass, Nucl. Phys. B123, 100 (1977).
[20] J. L. Gervais, A. Jevicki, and B. Sakita, Perturbation expansion around extended particle states in quantum field theory. 1., Phys. Rev. D 12, 1038 (1975).

[21] J. Evslin, The $\phi^{4}$ kink mass at two loops, arXiv:2104.07991.

[22] R. F. Dashen, B. Hasslacher, and A. Neveu, Nonperturbative methods and extended hadron models in field theory 2. Two-dimensional models and extended hadrons, Phys. Rev. D 10, 4130 (1974).

[23] A. B. Zamolodchikov, Mass scale in the Sine-Gordon model and its reductions, Int. J. Mod. Phys. A 10, 1125 (1995).

[24] A. R. Aguirre and G. Flores-Hidalgo, A note on one-loop soliton quantum mass corrections, Mod. Phys. Lett. A 35, 2050102 (2020).

[25] I. Takyi, M. K. Matfunjwa, and H. Weigel, Quantum corrections to solitons in the $\Phi^{8}$ model, Phys. Rev. D 102, 116004 (2020).

[26] M. Lizunova and J. van Wezel, An introduction to kinks in $\varphi^{4}$-theory, SciPost Phys. Lect. Notes 23, 1 (2021).

[27] C. Adam, K. Oles, T. Romanczukiewicz, and A. Wereszczynski, Kink-antikink collisions in a weakly interacting $\phi^{4}$ model, Phys. Rev. E 102, 062214 (2020).

[28] C. Halcrow, Quantum soliton scattering manifolds, J. High Energy Phys. 07 (2020) 182.

[29] A. Alonso-Izquierdo, L. M. Nieto, and J. Queiroga-Nunes, Scattering between wobbling kinks, Phys. Rev. D 103, 045003 (2021).

[30] I. V. Melnikov, C. Papageorgakis, and A. B. Royston, Accelerating solitons, Phys. Rev. D 102, 125002 (2020).

[31] I. Melnikov, C. Papageorgakis, and A. B. Royston, The Forced Soliton Equation and Semiclassical Soliton Form Factors, Phys. Rev. Lett. 125, 231601 (2020).

[32] A. Roy, D. Schuricht, J. Hauschild, F. Pollmann, and H. Saleur, The quantum sine-Gordon model with quantum circuits, arXiv:2007.06874.

[33] J. Y. Kim and B. D. Sun, Gravitational form factors of a baryon with spin-3/2, Eur. Phys. J. C 81, 85 (2021). 